Draft version December 11, 2019

Typeset using $\mathrm{LAT}_{\mathrm{E}} \mathrm{X}$ twocolumn style in AASTeX63

\title{
PopSyCLE: A New Population Synthesis Code for Compact Object Microlensing Events
}

\author{
Casey Y. Lam, ${ }^{1}$ Jessica R. Lu, ${ }^{1}$ Matthew W. Hosek, Jr.,${ }^{2}$ William A. Dawson,${ }^{3}$ and Nathan R. Golovich ${ }^{3}$ \\ ${ }^{1}$ University of California, Berkeley, Department of Astronomy, Berkeley, CA 94720 \\ ${ }^{2}$ University of California, Los Angeles, Department of Astronomy, Los Angeles, CA 90095 \\ ${ }^{3}$ Lawrence Livermore National Laboratory, 7000 East Ave., Livermore, CA 94550
}

(Received; Revised; Accepted)

Submitted to

\begin{abstract}
We present a new Milky Way microlensing simulation code, dubbed PopSyCLE (Population Synthesis for Compact object Lensing Events). PopSyCLE is the first resolved microlensing simulation to include a compact object distribution derived from numerical supernovae explosion models and both astrometric and photometric microlensing effects. We demonstrate the capabilities of PopSyCLE by investigating the optimal way to find black holes (BHs) with microlensing. Candidate BHs have typically been selected from wide-field photometric microlensing surveys, such as OGLE, by selecting events with long Einstein crossing times $\left(t_{E}>120\right.$ days). These events can be selected at closest approach and monitored astrometrically in order to constrain the mass of each lens; PopSyCLE predicts a BH detection rate of $\sim 40 \%$ for such a program. We find that the detection rate can be enhanced to $\sim 85 \%$ by selecting events with both $t_{E}>120$ days and a microlensing parallax of $\pi_{E}<0.08$. Unfortunately, such a selection criterion cannot be applied during the event as $\pi_{E}$ requires both pre- and post-peak photometry. However, historical microlensing events from photometric surveys can be revisited using this new selection criteria in order to statistically constrain the abundance of BHs in the Milky Way. The future WFIRST microlensing survey provides both precise photometry and astrometry and will yield individual masses of $\mathcal{O}(100-1000)$ black holes, which is at least an order of magnitude more than is possible with individual candidate follow-up with current facilities. The resulting sample of BH masses from WFIRST will begin to constrain the shape of the black hole present-day mass function, $\mathrm{BH}$ multiplicity, and BH kick velocity distributions.
\end{abstract}

\section{INTRODUCTION}

The Milky Way is predicted to contain $10^{8}-10^{9}$ stellar mass black holes (Agol \& Kamionkowski 2002); however, the exact number is still very uncertain. Only a few dozen black holes (BHs) have been detected to date in X-ray binaries or BH-BH mergers (Remillard \& McClintock 2006; Abbott et al. 2016); no isolated stellar mass BHs have yet been definitively and unambiguously detected. Although BHs likely form after the death of a massive star, uncertainties in the final stages of massive star evolution have made quantitative predictions of BH masses and numbers difficult (Heger et al. 2003). Whether a massive star will form a $\mathrm{BH}$, neutron star, or compact remnant at all is not only a function of the

Corresponding author: Casey Y. Lam

casey_lam@berkeley.edu initial stellar mass, but other factors such as metallicity, stellar winds and the core structure at time of collapse. Additionally, it is still unknown whether $\mathrm{BH}$ binary systems form directly from stellar binaries, dynamically from two isolated $\mathrm{BHs}$, or if there are multiple formation channels.

Detecting and characterizing a sample of isolated stellar mass BHs would provide insight into these unsolved problems by constraining the number of $\mathrm{BHs}$ in the Milky Way and the present-day mass function, binary fraction, and spatial and velocity distributions. This in turn will place constraints on stellar evolution models and improve understanding of supernova physics (Janka 2012). It would also allow better interpretation of recent LIGO results. For example, is the "mass gap" between the heaviest neutron stars and the lightest BHs real, or is it an artifact of the observational bias resulting from only having detected binary BHs (from an observational 
perspective, see Abbott et al. (2019); Özel et al. (2010); Farr et al. (2011); from a theoretical perspective, see Belczynski et al. (2012); Fryer et al. (2012))?

Gravitational microlensing is a technique particularly well suited for detecting dark isolated objects in the Milky Way such as BHs, as properties of the lens can be inferred from changes in the source image, without having to directly observe the lens itself (Paczynski 1986). For example, the MACHO (Alcock et al. 1993), EROS (Aubourg et al. 1993), and OGLE (Udalski et al. 1994) collaborations used gravitational microlensing to determine that the majority of dark matter in the Galactic halo is not due to ordinary baryonic matter (Alcock et al. 2000; Tisserand et al. 2007; Wyrzykowski et al. 2011). However, after the LIGO BH-BH merger detection, there has been a major resurgence in interest in $\mathrm{BHs}$ (and in particular primordial $\mathrm{BHs}$ ) as a viable dark matter candidate (Bird et al. 2016; Sasaki et al. 2016). Additionally, for the past 15 years microlensing has been used to detect extrasolar planets; for a review see Gaudi (2012). Currently, microlensing is being used to search for isolated BHs by combining information from the photometric brightening and parallax signal and the astrometric shift of the source image to constrain the mass of the lens (Lu et al. 2016; Rybicki et al. 2018). As all current microlensing surveys are ground-based photometric surveys, a substantial development is space-based survey telescopes such as Gaia and WFIRST, which will provide astrometric measurements for microlensing events (Gaia Collaboration et al. 2016; Rybicki et al. 2018; Spergel et al. 2015; Penny et al. 2019).

Previous theoretical work modeled microlensing in the Milky Way due to a population of stars, brown dwarfs, and stellar remnants (Han \& Gould 2003; Wood \& Mao 2005). However, these models use a heavily simplified model for compact object population synthesis. Osłowski et al. (2008) investigated compact object lensing by using the StarTrack population code (Belczynski et al. 2008) to generate isolated BHs and neutron stars via two different formation channels. However, in all these models a realistic extinction map, which varies significantly across the sky and affects both the optical depth and events rates, was lacking. Dai et al. (2015) followed Wood \& Mao (2005) to specifically investigate lensing by neutron stars, but incorporated updated kinematic information and extinction via a variable luminosity function, showing that most neutron star lensing events occur on much shorter timescales than previously though, and that the steepness of the luminosity function has a strong effect on the timescale distribution. However, they did not have updated modeling for BHs.
Several new simulations have been recently developed to investigate optical depths and event rates of Galactic microlensing involving more sophisticated Galactic models and realistic extinction (Penny et al. 2013; Awiphan et al. 2016). However, the population synthesis in these models lack high-mass stellar remnants as they are primarily focused on lower mass stars and exoplanets.

In this paper, we will describe new a code we have developed called Population Synthesis for Compact object Lensing Events (PopSyCLE). ${ }^{1}$ White dwarfs, neutron stars, and BHs are synthesized and injected into a stellar model of the Milky Way using stellar evolution models and an initial-final mass relation. Each individual object is then propagated in time to perform a synthetic microlensing survey. Cuts are then made on observational quantities. Since the simulation is resolved, all microlensing parameters are known for each individual event. We can then begin to investigate microlensing events due to $\mathrm{BH}$ lenses.

The rest of the paper is organized as follows. In $\S 2$, the input models used to perform both stellar and compact object population synthesis in PopSyCLE are described. The details of the compact object population synthesis are given in $\S 3$, and the procedure for selecting microlensing events is given in $\S 4$. Simulation parameters are presented in $\S 5$, then synthetic PopSyCLE surveys are compared to observations and theory in $\S 6$. In $\S 7$ we consider strategies for $\mathrm{BH}$ microlensing candidate selection, search, and verification, both from the ground and with the upcoming WFIRST mission. A discussion of PopSyCLE compared to other work is presented in $\S 8$, along with comments on further developments to be made to the code. Lastly, in $\S 9$ we present our conclusions.

\section{POPSYCLE INGREDIENTS}

To calculate microlensing event rates and parameters, knowledge of the masses, positions, and velocities of stars and compact objects in the Milky Way is required. Photometric information to estimate quantities such as blending and survey depth limits is also required. We utilize existing models of the Milky Way to describe the stars $(\S 2.1)$ and inject compact objects according to our own population synthesis estimates and an initial-final mass relation for BHs, neutron stars, and white dwarfs $(\S 2.2)$.

\subsection{Generating Stars with Galaxia}

Galaxia is a synthetic stellar survey of the Milky Way (Sharma et al. 2011). Given a survey area and location,

${ }^{1}$ https://github.com/jluastro/PopSyCLE 


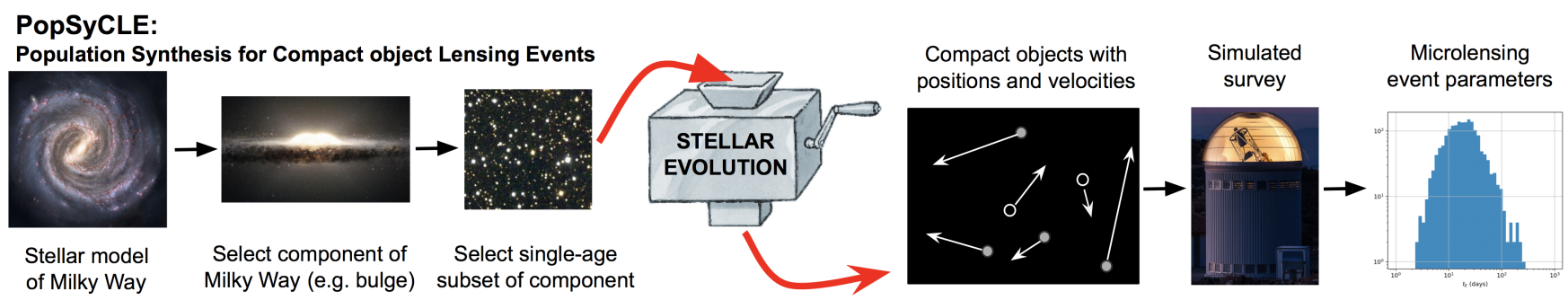

Figure 1. A schematic of the PopSyCLE pipeline. The first three panels are described in $\S 2.1$. The next two panels are described in $\S 2.2$ and 3. The last two panels are described in $\S 4$. Image credits: Universe Today (Panel 1), ESO/NASA/JPL-Caltech/M. Kornmesser/R. Hurt (Panel 2), NOAO/AURA/NSF (Panel 3), Simply scheme: introducing computer science, Harvey and Wright (Panel 4), OGLE survey (Panel 6).

Galaxia will generate a stellar model of the Milky Way for a circular field of sky, which corresponds to a 3-D conical volume. Galaxia is a resolved simulation; that is, for every single star in the field, it will return a position, velocity, age, mass, photometry in several filters, 3-D extinction, metallicity, surface gravity, and more. ${ }^{2}$ Galaxia implements the Besançon analytic model for the Milky Way (Robin et al. 2003), with a modified version of the disk kinematics that adjusts the velocity in the azimuthal direction (Shu 1969). For a detailed description of the implementation and galactic model parameters see Sharma et al. (2011) and references therein; for a summary of the model, see Tables 1-3 in Sharma et al. (2011). We also perform additional modifications to Galaxia's bulge kinematics, as they are known to produce some inconsistencies with observations. This is described in more detail in Appendix A. In the rest of the main text, whenever reference is made to Galaxia, we specifically mean Galaxia with the modified bulge, unless otherwise mentioned. Additionally, there is also an option to implement the N-body stellar halo model of Bullock \& Johnston (2005) instead of the smooth analytic stellar halo model from the Besançon model. We choose to use the smooth analytic model instead of the N-body model as we are primarily considering lensing in the disk and bulge; stellar halo substructure is not a significant concern.

Galaxia includes a 3-D extinction map; however, the built-in extinction law from Schlegel et al. (1998) is not well suited to the high-extinction regions towards the inner Galactic bulge. Unfortunately, the newest 3-D extinction maps (e.g. Green et al. (2018)) do not cover significant fractions of the Galactic Bulge region. Similarly, the extinction maps of Nataf et al. (2013), which were constructed using OGLE, VVV, and 2MASS data, only cover the OGLE-III bulge fields. Instead, we modify the Galaxia output in order to adopt the reddening

\footnotetext{
2 http://galaxia.sourceforge.net/Galaxia3pub.html
}

law of Damineli et al. (2016) as described in Appendix B.

\subsection{Generating Compact Objects}

PyPopStar is a software package that generates singleage, single-metallicity populations (i.e. star clusters) using adjustable initial mass functions (IMFs), multiplicity distributions, stellar evolution and atmosphere grids, and extinction laws; for a more detailed description see $\S 4$ of Hosek et al. (2019). ${ }^{3}$ In summary, IMFs are parametrized as piecewise functions consisting of multiple power-laws and generated according to the prescription from Pflamm-Altenburg \& Kroupa (2006). We adopt the MESA Isochrones and Stellar Tracks (MIST) v1.2 stellar evolution models (Choi et al. 2016; Dotter 2016), which are calculated using the Modules for Experiments in Stellar Astrophysics (MESA) code (Paxton et al. 2011, 2013, 2015). These models provide theoretical isochrones with stellar temperatures and surface gravities as a function of mass at a given stellar age. Model atmosphere grids are then used to assign an atmosphere to each star. The stellar temperature determines which grid is used; an ATLAS9 grid is used for stars with $\mathrm{T}_{\text {eff }}>5500 \mathrm{~K}$ (Castelli \& Kurucz 2004), a PHOENIX grid is used for $3800 \mathrm{~K}<\mathrm{T}_{\text {eff }}<5000 \mathrm{~K}$ (version 16; Husser et al. 2013), and a BTSettl grid is used for $1200 \mathrm{~K}<\mathrm{T}_{\text {eff }}<3200 \mathrm{~K}$ (Baraffe et al. 2015). For temperatures at a transition region between grids (e.g. $5000 \mathrm{~K}-5500 \mathrm{~K}$ ), an average atmosphere between the two model grids is used. For all models, solar metallicity is assumed. This assumption will not have a large effect on the $\mathrm{BH}$ population synthesis; see $\S 8.4 .2$ for justification. Although the MIST stellar evolution models do not evolve stars all the way down to neutron stars and $\mathrm{BHs}$, it does have some partial support for white dwarfs; a full discussion of stellar evolution with white dwarfs is presented in $§ 2.2 .2$.

\footnotetext{
${ }^{3}$ https://github.com/astropy/PyPopStar
} 
The PyPopStar software as described in $\S 4$ of Hosek et al. (2019) would discard stars that had evolved into compact objects. To be able to perform population synthesis, we have updated PyPopStar with an initialfinal mass relation (IFMR) that will properly account for compact objects. Given the zero-age main sequence (ZAMS) mass of a star, the IFMR describes the type and mass of compact object formed. The IFMR is an active area of research, as the ZAMS mass does not solely determine the evolutionary path of a star. Factors such as rotation (which induces mixing in the star) and mass loss due to stellar winds (which are a function of metallicity) are important in the final stages of stellar evolution. In particular, the pre-supernova core structure of the star strongly determines whether there is an explosion or not, which in turn affects the type of compact remnant formed (Sukhbold et al. (2018) and references therein). We modify PyPopStar to implement a combination of IFMRs taken from recent simulations to build a population of white dwarfs, black holes, and neutron stars as described in more detail below.

Note that PyPopStar has support for stellar multiplicity. However, as Galaxia only has a treatment of single stars, this functionality was not used. A discussion of this is presented in $\S 8.4 .1$.

\subsubsection{Neutron Stars and Black Holes}

For neutron stars (NSs) and BHs, we have adopted the IFMR presented in Raithel et al. (2018), hereafter R18, which is calculated by combining observational data of $\mathrm{BH}$ and NS masses along with 1-D neutrinodriven supernova simulations (Sukhbold et al. 2016). We have made several minor modifications, described in Appendix C. The IFMR of R18 is only a function of the ZAMS mass; other factors that influence a $\mathrm{BH}$ or NS outcome such as small differences in core structure are taken into account by making the IFMR probabilistic. For a given ZAMS mass, the probability of forming a $\mathrm{BH}$ or a NS is given in Table 1; the values come from R18. There are then two separate IFMRs for BHs and NSs. The BH IFMR is a piecewise function (Figure 7.1), while we make the assumption that the NS IFMR is a constant (justification in Appendix C.) Thus, because of the probabilistic nature of the total IFMR, one ZAMS mass can be mapped to two remnant masses, of either a $\mathrm{BH}$ or NS.

The maximum $\mathrm{BH}$ mass generated by the $\mathrm{BH}$ IFMR is about $16 M_{\odot}$. This appears to run contrary to the recent results from gravitational wave astronomy experiments, which has found 15 out of 20 pre-merger black holes with masses above $16 M_{\odot}$ (The LIGO Scientific Collaboration et al. 2018). As discussed in detail in $\S 6$ of R18, the models of Sukhbold et al. (2016) are all solar metallicity, while the formation of LIGO-mass BHs from single-star evolution requires lower metallicity. Additionally, the models are of single stars, and do not consider formation of BHs via dynamical assembly in dense stellar clusters nor strong magnetic fields, other proposed mechanism for obtaining LIGO-mass BHs. See $\S 8.4 .2$ and $\S 8.4 .1$ for a further discussion.

\subsubsection{White Dwarfs}

One complicating factor in white dwarf (WD) population synthesis is that the MIST models do not include the late stages of WD cooling. Thus not all WDs are generated, in particular the oldest and faintest ones. This means a WD IFMR is also required. For all white dwarfs not included in a set of evolutionary models, we use the empirically determined IFMR of Kalirai et al. (2008), hereafter K08, given by

$$
M_{W D}=\left(0.109 M_{Z A M S}+0.394\right) M_{\odot} .
$$

The data in K08 range in initial mass from $1.16 M_{\odot}<$ $M_{Z A M S}<6.5 M_{\odot}$. However, to have continuous coverage in $M_{Z A M S}$ for the IFMR, we extend the domain to $M_{Z A M S}$ to $0.5 M_{\odot}<M_{Z A M S}<9 M_{\odot}$, where the lower mass limit was chosen the match the MIST models' lower mass limit, and the upper mass limit was chosen to match where the IFMR for NSs and BHs begin. The low-mass extrapolation covers all possible WDs formed within the age of the Universe. The high-mass extrapolation ensures that that there is an IFMR for all stellar masses and the maximum WD mass would then be

$$
M_{W D}\left(M_{Z A M S}=9 M_{\odot}\right)=1.375 M_{\odot} \lesssim M_{c h}
$$

where $M_{c h}=1.4 M_{\odot}$ is the Chandrasehkar mass.

In particular for the MIST models, WDs are tracked only so far down the cooling curve and the coolest/oldest WDs are usually dropped. For these objects, the WD IFMR (Equation 1) is used to produce dark WDs and their luminosity is neglected. This is justified as these old WDs have absolute magnitudes of $\mathrm{M} \sim 18$ in redoptical filters and they contribute negligible flux at typical distances for lenses and sources (e.g. Campos et al. 2016). Thus a PopSyCLE population will contain both luminous WDs from the MIST models and dark WDs from the K08 IFMR.

\section{POPULATION SYNTHESIS}

First, Galaxia is used to generate a synthetic stellar survey of a circular area on the sky. Only stars that are within a distance of $20 \mathrm{kpc}$ to Earth are kept, as these are the ones most likely to be observable lenses 
Table 1. Compact Object Formation Probabilities

\begin{tabular}{rcccc}
\hline \hline Mass & Range $\left(M_{\odot}\right)$ & $P_{W D}$ & $P_{N S}$ & $P_{B H}$ \\
\hline 0.5 & $<\mathrm{M}<9$ & 1.000 & 0 & 0 \\
9 & $<\mathrm{M}<15$ & 0 & 1.000 & 0 \\
15 & $<\mathrm{M}<17.8$ & 0 & 0.679 & 0.321 \\
$17.8<\mathrm{M}<18.5$ & 0 & 0.833 & 0.167 \\
18.5 & $<\mathrm{M}<21.7$ & 0 & 0.500 & 0.500 \\
21.7 & $<\mathrm{M}<25.2$ & 0 & 0 & 1.000 \\
25.2 & $<\mathrm{M}<27.5$ & 0 & 0.652 & 0.348 \\
27.5 & $<\mathrm{M}<60$ & 0 & 0 & 1.000 \\
60 & $<\mathrm{M}<120$ & 0 & 0.400 & 0.600 \\
\hline
\end{tabular}

Note - Given the ZAMS mass (the first column), what are the probabilities of forming a white dwarf, neutron star, or black hole. The NS and BH columns come from Table 3 of R18.

and sources. In addition to mass, age, 6-D kinematics, and photometry, Galaxia also tags each star by its population type (thin disk, thick disk, bulge, stellar halo.) The stars generated are sorted by population to preserve age and kinematic information. The thin disk is a multi-age population ranging from less than $0.15 \mathrm{Gyr}$ up to 10 Gyr; we split the thin disk population into subpopulations of 29 finer age bins. The thick disk, bulge, and halo are all single-age populations of 11,10 , and 14 Gyr, respectively. Some of the youngest stars in the thin disk need to have their ages reassigned because the youngest age available for the MIST isochrones is $10^{5}$ years. This is only necessary for a very small number of stars; for example, in a survey pointed toward the Galactic center, less than 0.001 percent of stars were younger than $10^{5}$ years. Additionally, the age of the 14 Gyr halo star population are reassigned to be $13.8 \mathrm{Gyr}$, to be within the age of the universe.

Each of these (sub)-populations is then approximated as a group of single-age stars. If necessary, these age groups are further broken up into smaller sub-groups of 2 million stars each, to keep the population synthesis calculation manageable.

Knowledge of the initial mass of each age group of stars is needed to perform the compact object population synthesis. The present-day mass of the age group can be calculated with knowledge of the ZAMS mass of each star. The initial age group mass can be found by multiplying the present-day group mass by an initialcurrent mass ratio factor; the calculation of this ratio is described in Appendix D. With the initial mass of the age group of stars in hand, we can then generate a similar group of stars of the correct mass and age with PyPopstar.

Next, compact objects are generated to inject back into the stellar population using PyPopStar. A Kroupa $\operatorname{IMF} \xi(m)=d N / d m \propto m^{-\alpha}$,

$$
\alpha= \begin{cases}-1.3, & 0.1 M_{\odot}<m \leq 0.5 M_{\odot} \\ -2.3, & 0.5 M_{\odot}<m \leq 120 M_{\odot}\end{cases}
$$

is assumed with the lower mass limit set to match the MIST models' lower mass limit, and the upper mass limit set to match the IFMR's upper mass limit (Kroupa 2001). Any object that evolves off the isochrone becomes a compact object. As described in $\S 2.2$, the IFMRs of R18 and K08 are used to obtain masses for BHs, NSs, and WDs.

We assume the positions and velocities of the compact objects follows that of the stellar population in Galaxia; they are assigned using kernel density estimation (KDE). The 6-D kinematic data is simultaneously fit using a Gaussian kernel and Euclidean metric with a bandwidth of 0.0001. Such a small bandwidth means the data is extremely unsmoothed. However, this is desired as we want to randomly draw from the existing distribution. For the BHs and NSs, a tuneable birth kick is also applied in a random direction in addition to the stellar velocity assigned from KDE. NSs have been observed to have birth velocities of $\sim 200-500 \mathrm{~km} / \mathrm{s}$, and up to 1000 $\mathrm{km} / \mathrm{s}$; it is thought that the initial kick velocity is due to asymmetry in the supernova explosion (Lai 2001; Janka 2012). BHs should also receive kicks; by conservation of momentum, BH kicks should be smaller than NS kicks (although see Janka (2013); Repetto et al. (2012) which suggests they will be of similar magnitude).

All the compact objects produced with the IFMRs are assumed to be dark, while luminous WDs produced with the MIST models are assigned the same color excess $E(B-V)$ as the star nearest to them in 3-D space.

For easier processing and parallelization, the stars from Galaxia and the compact objects generated from PyPopStar are sorted into a grid of Galactic latitude and longitude bins as seen from the solar system barycenter. The size of these latitude and longitude bins $\Delta \theta_{\text {bin }}$ must be sufficiently large such that most stars do not cross over multiple bins during the survey duration, but not so large that finding source-lens pairs is computationally infeasible (described in $\S 4.3$ ). Assuming that typical microlensing surveys are of $\sim 5$ years, and proper motions of $>5 \mathrm{arcsec} /$ year are rare, this gives a lower limit on $\Delta \theta_{\text {bin }}$ of $25^{\prime \prime}$. We have chosen the default bin sizes to be roughly $30^{\prime \prime} \times 30^{\prime \prime}$; however, the bin size is adjustable by the user. 
The above process of generating compact objects and sorting them into bins for each (sub)-population group is then repeated. Thus, the generated compact objects preserve the correlations between population age, structure, and dynamics that are present in the stellar population, with the addition of tunable birth kicks.

\section{IDENTIFYING MICROLENSING EVENTS}

Microlensing occurs when a foreground star or compact object (the lens) passes near a background star (the source) as projected on the sky. However, not all close approaches may produce detectable or significant microlensing events. Therefore, microlensing event candidates are first identified, then detailed properties of the lensing event are calculated and used to decide whether the candidate is significant enough to classify as a microlensing event. In $\S 4.1$ and 4.2 , basic definitions for microlensing event parameters are presented. The process by which candidates are identified and converted into a final microlensing list is described in $\S 4.3$ and 4.4. Going forward, the point source point lens (PSPL) regime of microlensing is assumed.

\subsection{Candidate Selection Parameters}

The scale of the microlensing event is set by the the angular Einstein radius

$$
\theta_{E}=\sqrt{\frac{4 G M}{c^{2}}\left(\frac{1}{d_{L}}-\frac{1}{d_{S}}\right)},
$$

where $d_{S}$ is the observer-source distance, $d_{L}$ is the observer-lens distance, and $M$ is the mass of the lens. Note that in microlensing, $\theta_{E}$ is unresolved, unlike in strong lensing. For a "typical" microlensing event consisting of a stellar bulge source at $d_{S}=8 \mathrm{kpc}$ and a stellar disk lens at $d_{L}=4 \mathrm{kpc}$ with a mass $M=0.5 M_{\odot}$, the Einstein radius is $\theta_{E}=0.71$ mas. For context, the highest-resolution images from the largest ground-based or space-based telescopes (with a filled aperture) is 50 mas in the optical or infrared. However, in some cases Einstein radii of this scale are interferometrically resolvable (Dong et al. 2019), moving from the regime of microlensing to strong lensing.

The Einstein crossing time $t_{E}$, the time it takes for the lens to traverse the Einstein radius, can be inferred from the photometric light curve. The magnitude of the relative source-lens proper motion $\mu_{\text {rel }}$ relates the Einstein crossing time and Einstein radius via

$$
\theta_{E}=\mu_{r e l} t_{E}
$$

The dimensionless source-lens separation vector normalized in units of the Einstein radius is

$$
\vec{u}=\frac{\vec{\theta}_{S}-\vec{\theta}_{L}}{\theta_{E}},
$$

where $\vec{\theta}_{L}$ and $\vec{\theta}_{S}$ denote the angular position of the lens and source on the sky, respectively. The magnitude of the separation $|\vec{u}|=u$ as a function of time, neglecting higher-order effects such as parallax, can be written as

$$
u(t)=\sqrt{u_{0}^{2}+\left(\frac{t-t_{0}}{t_{E}}\right)^{2}},
$$

where $t_{0}$ is the time of closest approach and $u_{0}$ is the separation at $t_{0} ; u_{0}$ is also known as the impact parameter.

The photometric amplification of the source is given by

$$
A(u)=\frac{u^{2}+2}{u \sqrt{u^{2}+4}},
$$

and is maximized at $u=u_{0}$. It can be seen from this formula that a smaller $u_{0}$ produces a larger amplification. Most microlensing surveys define a "microlensing event" as an event that has $u_{0}$ less than 1 or 2 .

The source flux fraction, which is sometimes called the blend parameter, or confusingly, the blend fraction, is the fraction of source flux over total flux in the telescope's photometric extraction aperture

$$
b_{S F F}=\frac{F_{S}}{F_{S}+F_{L}+F_{N}},
$$

where $F_{S}, F_{L}$, and $F_{N}$ are the fluxes due to the (unlensed) source, lens, and any neighboring stars. From this definition, a highly blended event will have a source flux fraction of $b_{S F F} \approx 0$ and an event with no blending (i.e. only flux from the source) will have $b_{S F F}=1$.

The photometric extraction aperture is typically proportional to the spatial resolution of the images; we adopt an aperture diameter of the full-width halfmaximum (FWHM), which is set by seeing for groundbased surveys like OGLE and the diffraction limit for space-based surveys such as WFIRST (these surveys are described in $§ 5)$. To give concrete numbers, the median seeing for the OGLE survey is roughly 1.3", which we take to be the FWHM. WFIRST, a planned 2.4 meter space telescope observing at $1630 \mathrm{~nm}$ (H-band), will have FWHM $\sim 0.17^{\prime \prime}$. These FWHM values for OGLE and WFIRST would correspond to aperture radii of roughly $0.65^{\prime \prime}$ and $0.09^{\prime \prime}$, respectively.

The bump magnitude, $\Delta m$, is the difference between the baseline magnitude $m_{\text {base }}$ and the peak magnitude $m_{\text {peak }}$

$$
\Delta m=-2.5 \log _{10}\left(\frac{A\left(u_{0}\right) F_{S}+F_{L}+F_{N}}{F_{S}+F_{L}+F_{N}}\right) .
$$


Note that although the magnification of the source flux is achromatic, the bump magnitude and the source flux fraction are dependent on wavelength and the photometric extraction aperture (Di Stefano \& Esin 1995). Thus, when events are selected on $\Delta m$ or $b_{S F F}$, we explicitly note the filter and aperture used.

\subsection{Other Selection Parameters}

In addition to the selection parameters described in $\S 4.1$, there are a number of other measureable microlensing quantities. In particular, the astrometric and microlensing parallax signals are useful for identifying possible black hole microlensing events.

The astrometric shift $\vec{\delta}_{c}$ of the source image centroid, assuming no blending, is given by

$$
\vec{\delta}_{c}=\frac{\theta_{E}}{u^{2}+2} \vec{u} .
$$

In contrast to the photometric amplification, the maximum astrometric shift occurs at $u= \pm \sqrt{2}$. Thus, if $u_{0}<\sqrt{2}$, the maximum astrometric shift will occur before and after the maximum photometric amplification. However, if $u_{0} \geq \sqrt{2}$, the time at which maximum astrometric shift and photometric amplification occur will coincide. It should be noted that $\delta_{c}$ is the maximum possible astrometric shift for a given geometry (i.e. for given $\left.M, d_{L}, d_{S}\right)$, as it does not include blending.

Blending due to a luminous lens modifies the astrometric signal to

$$
\vec{\delta}_{c, L L}=\frac{\theta_{E}}{1+g} \frac{1+g\left(u^{2}-u \sqrt{u^{2}+4}+3\right)}{u^{2}+2+g u \sqrt{u^{2}+4}} \vec{u}
$$

where $g=F_{L} / F_{S}$ (Dominik \& Sahu 2000). Blending due to the lens and other neighboring stars dilutes the astrometric signal further to

$$
\vec{\delta}_{c, L L N}=\frac{\tilde{A}(u) F_{S} \vec{\theta}_{S}+F_{N} \vec{\theta}_{N}}{A(u) F_{S}+F_{L}+F_{N}}-\frac{F_{S} \vec{\theta}_{S}+F_{N} \vec{\theta}_{N}}{F_{S}+F_{L}+F_{N}},
$$

where $\vec{\theta}_{S}$ and $\vec{\theta}_{N}$ are the angular positions of the source and the centroid of all the on-sky neighbors contributing to blending, relative to the lens position; $A(u)$ is given by Equation 7 and $\tilde{A}(u)$ is defined

$$
\tilde{A}(u)=\frac{u^{2}+3}{u \sqrt{u^{2}+4}} .
$$

For long-duration events ( $t_{E} \gtrsim 3$ months), the motion of Earth orbiting the Sun will modify the otherwise symmetric light curve. This signal is called the microlensing parallax $\pi_{E}$ and is given by

$$
\pi_{E}=\frac{\pi_{r e l}}{\theta_{E}},
$$

where $\pi_{r e l}$ is the relative parallax

$$
\pi_{r e l}=1 \mathrm{AU}\left(\frac{1}{d_{L}}-\frac{1}{d_{S}}\right) .
$$

Combining Equations 3 and 15 and solving for the lens mass $M$ yields

$$
M=\frac{\theta_{E}}{\kappa \pi_{E}},
$$

where $\kappa \equiv \frac{4 G}{1 A U \cdot c^{2}}$. If both the photometric magnification $A(u)$ and the astrometric shift $\delta_{c}\left(u, \theta_{E}\right)$ can be measured, the Einstein radius $\theta_{E}$ can be deduced. Along with a measurement of the microlensing parallax $\pi_{E}$, the mass of the lens $M$ can then be derived using Equation 16. Thus, by having both astrometric and photometric measurements, the degeneracies between lens mass, lens distance, and source distance can be broken. ${ }^{4}$ The lens mass can then be constrained without having to resort to assumptions about the lens and source distances. Many microlensing studies only measure the photometric signal and use a Galactic model to weight different source distances in order to derive the lens mass; however, this is a significant assumption. By combining both the photometric and astrometric microlensing signal, the lens mass can be determined without having to resort to modeling distances. Additionally, the astrometric signal peaks after the photometric signal when $u_{0}<\sqrt{2}$.

The method just described can be used to measure the masses of BHs. Since long-duration microlensing events are more likely to be $\mathrm{BHs,}$, probable $\mathrm{BH}$ microlensing events can be selected from ground-based photometry, then followed up astrometrically (Lu et al. 2016).

\subsection{Event Selection Algorithm}

With population synthesis complete, we can specify a duration and sampling cadence to perform a synthetic microlensing survey. Typical microlensing survey lengths are on the order of years, over which celestial motions are linear for nearly all lenses and sources. Thus, with an initial position and velocity, the position of any object at some later time in the survey can be calculated.

First, a grid of latitude and longitude bins is overlaid across the entire survey area. Next, four adjacent latitude and longitude bins of objects generated from population synthesis are read in and combined to make one

\footnotetext{
${ }^{4}$ In the case of more complicated microlensing scenarios (e.g. binary sources or lenses that may also be rotating), additional information might be needed to break new degeneracies introduced, such as incorporating e.g. spectroscopic measurements (Smith et al. 2002). Fitting and extracting masses from these types of events is beyond the scope of this paper, although we do discuss binary effects in $\S 8.4 .1$.
} 
a)

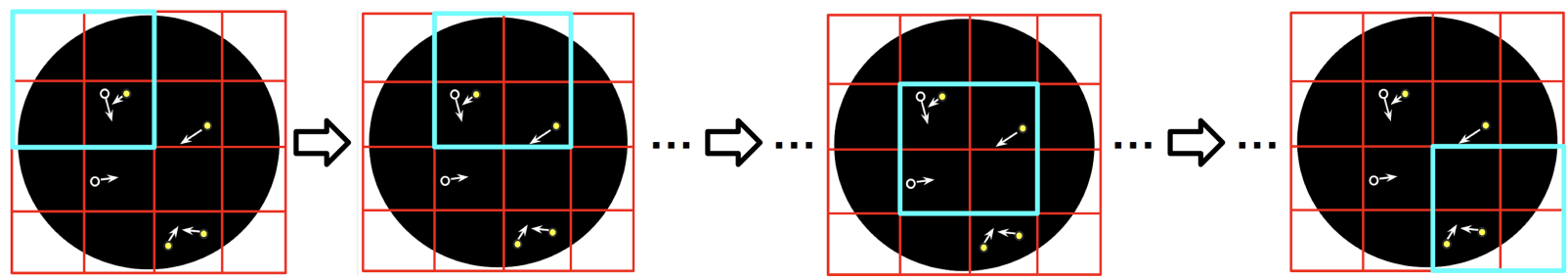

b)

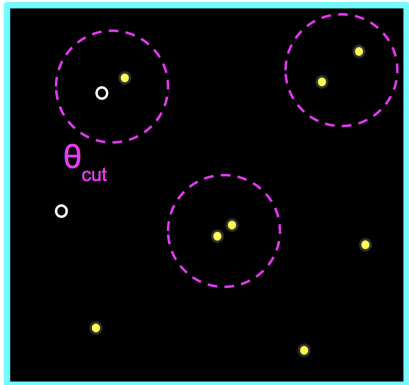

c)

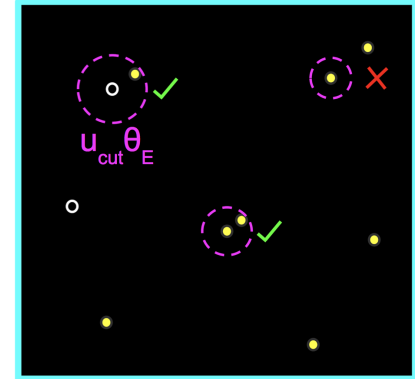

d)

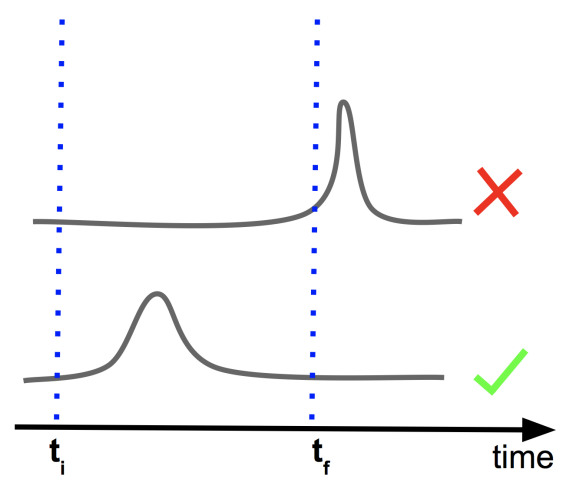

Figure 2. a) A schematic of the binning algorithm described in the second paragraph of $\S 4.3$. Each field is divided into a grid (red lines) and then the four adjacent boxes at each grid vertex are binned (blue square) to search for candidate microlensing events and contaminating neighbor stars. Within each blue bin, event selection criteria are applied (subfigures b through d). The process is then repeated on the next blue bin until the entire grid has been covered. Finally, results are aggregated and duplicates are removed. b) A schematic of Cut 1 , which selects all object pairs within some on-sky separation of $\Delta \theta<\theta_{\text {cut }}$. Microlensing parameters are computed for all of these object pairs. c) A schematic of Cut 2, which selects all of the objects pairs left after Cut 1 that satisfy the condition $\Delta \theta / \theta_{E}<u_{c u t}$. Note that unlike in Cut 1 , the circles have different radii, as $\theta_{E}$ depends on the lens mass and the source and lens distances. The pairs that pass this selection have green checks next to them, while those that do not pass the selection have a red " $\mathrm{x}$ ". d) A schematic of Cut 3, which selects the source-lens pairs whose point of closest approach on-sky occurs within the survey window. This is equivalent to selecting lightcurves that peak photometrically within the survey window. Note: none of these cartoons are to scale.

large bin over which microlensing events are searched for at the sampled times within the survey window. This combination ensures that microlensing events that occur across the edges of two smaller bins are not missed. The algorithm for finding event candidates has four "cuts" as follows:

1. Find the nearest on-sky neighbor for each object and calculate the separation $\Delta \theta$. Pairs with separations of $\Delta \theta>\theta_{\text {cut }}$ are rejected, where $\theta_{\text {cut }}$ is a user-specified separation. Analogous to the bin size selection, choosing too small a value for $\theta_{\text {cut }}$ will mean some events are missed. Choosing too large a value for $\theta_{\text {cut }}$ is less problematic; it will merely slow down subsequent calculations by considering pairs that will not produce a detectable microlensing signal.

2. Calculate the Einstein radius $\theta_{E}$ of the lenses from these initial microlensing candidate pairs. Only keep the pairs where $\Delta \theta / \theta_{E}<u_{c u t}$, where $u_{c u t}$ is another user-specified value that sets the maximum impact parameter. For this further refined list of candidates, record all the stars that fall within the seeing disk radius $\theta_{\text {blend }}$, which will be used to calculate blending later on. The seeing disk radius is also a user-specified parameter.

3. From the new list of microlensing candidate pairs, calculate the time of closest approach $t_{0}$ when $u=$ $u_{0}$. Events are kept if $t_{0} \in\left[t_{i}, t_{f}\right]$ where $t_{i}$ and $t_{f}$ are the start and end times for the survey.

4. Remove unphysical "events" where the source is a dark object.

This yields the list of microlensing event candidates. The procedure is then repeated for all the bins. This algorithm will generate many repeats, due to the multiple overlaps in the bins, and also the multiple evaluation times. Only unique events are counted, that is, a lenssource pair is only counted once; the event parameters that get recorded are the ones corresponding to when the source and lens are closest to each other. A cartoon schematic of this entire process is shown in Figure 4.3. 


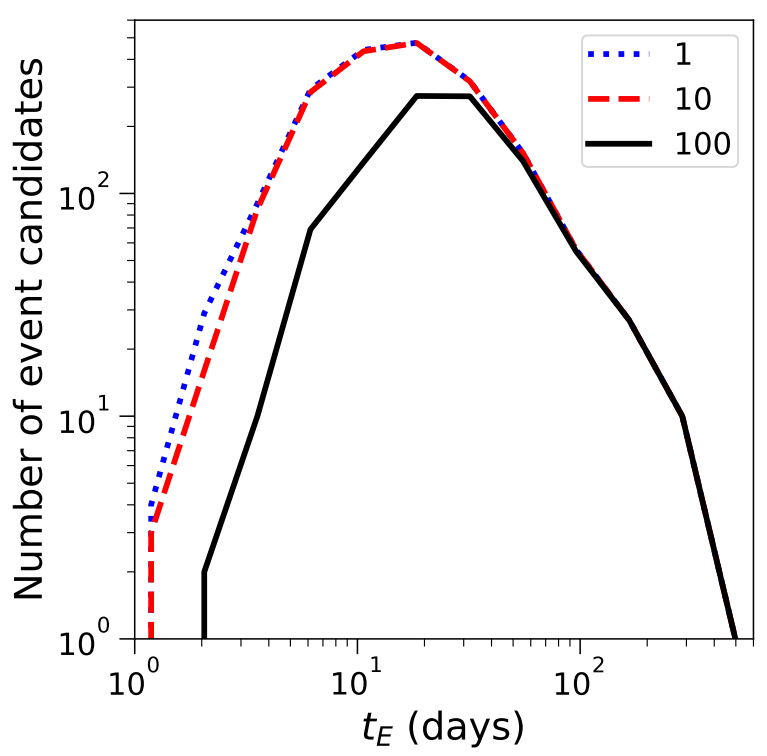

Figure 3. The $t_{E}$ distribution for all event candidates (i.e. no observational cuts) from field F01, but with three different survey cadences. The number in the legend corresponds to the sampling cadence, in days. The long timescale end above 30 days is insensitive to the sampling cadence. Cadences of 10 days or less provide an accurate description of the peak of the $t_{E}$ distribution.

With complete information about the 6-D kinematics, masses, and photometry, any microlensing parameters of interest can be calculated for all event candidates.

It should also be noted that the choice of sampling cadence $t_{o b s}$, defined as the time between consecutive observations, will change the number of event candidates. For example, $t_{o b s}=1$ day corresponds to a very dense sampling, while $t_{o b s}=100$ days corresponds to a very sparse sampling. There will be a loss of sensitivity to events with $t_{E}<t_{o b s}$ since shorter events will fall between observations. It should also be emphasized that the sampling cadence of PopSyCLE has no relation to the observational survey cadence of real microlensing surveys. For example, as shown in Figure 4.3, PopSyCLE run with a sampling cadence of 100 days finds nearly all events with $t_{E} \gtrsim 30$ days since objects only have to approach each other within $u_{c u t} \theta_{E}$ to be detected.

\subsection{Survey Implementation}

Many of the microlensing events reported by PopSyCLE as described in $\S 4.3$ would be undetectable by a real survey. For example, there will be events too faint to be observed. Thus, we consider the list of events generated in $\S 4.3$ to be event candidates. The user can select from this list of event candidates those which sat-
Table 2. Common Simulation Parameters

\begin{tabular}{|c|c|}
\hline Parameter & Value \\
\hline NS kick velocity & $350 \mathrm{~km} / \mathrm{s}$ \\
\hline BH kick velocity & $100 \mathrm{~km} / \mathrm{s}$ \\
\hline Duration & 1000 days \\
\hline Cadence & 10 days \\
\hline$\theta_{\text {cut }}$ & $2^{\prime \prime}$ \\
\hline$u_{\text {cut }}$ & 2 \\
\hline Extinction Law & Damineli et al. (2016) \\
\hline Area Per Field & $0.34 \mathrm{deg}^{2}$ \\
\hline \multicolumn{2}{|c|}{$\begin{array}{l}\text { NoTE-As described in } \S 3 \text {, a birth kick is added } \\
\text { to the progenitor star's velocity in a random di- } \\
\text { rection for black holes and neutron stars. As de- } \\
\text { scribed in } \S 4.3 \text {, duration describes the length of } \\
\text { synthetic survey, and cadence specifies the sam- } \\
\text { pling frequency. } \theta_{c u t} \text { is used to set the maximum } \\
\text { separation between potential microlensing event } \\
\text { candidates. Considering relatively extreme val- } \\
\text { ues to maximize } \theta_{E} \text {, such as } d_{L}=10 \mathrm{pc}, d_{S}=20 \\
\text { kpc, and } M=15 M_{\odot} \text { correspond to } \theta_{E}=0.11^{\prime \prime} \text {; } \\
\text { thus, the choice of } 2^{\prime \prime} \text { will capture all observ- } \\
\text { able microlensing events in the simulation. } u_{c u t} \\
\text { defines the maximum allowed } u_{0} \text {. }\end{array}$} \\
\hline
\end{tabular}

isfy some observational criteria to generate a final list of microlensing events.

\section{POPSYCLE SIMULATIONS}

All the event candidates generated by PopSyCLE are microlensing events, as there are no other types of transient phenomena (e.g. variable stars, telescope artifacts) in the simulation. Nonetheless, observational cuts are applied to replicate what a real survey would see. As different microlensing surveys use different telescopes, selection criteria, reduction pipelines and methods, etc. the cuts are different for each survey. We simulate several current and future microlensing surveys, as described below.

First, we provide background information on current surveys we will emulate. The Optical Gravitational Lensing Experiment (OGLE) (Udalski et al. 1992) and the Microlensing Observations in Astrophysics (MOA) (Muraki et al. 1999) collaborations run dedicated longterm photometric surveys monitoring the Milky Way bulge (and other targets) for microlensing events from small ground-based, seeing-limited telescopes. For instance, OGLE-IV regularly observes $\gtrsim 150 \mathrm{deg}^{2}$ of the Galactic bulge for 8 to 9 months out of the year, from February to October. The observing cadence typically ranges from $20 \mathrm{~min}$ to 2 days, depending on the sky 
Table 3. PopSyCLE Fields

\begin{tabular}{lrrl}
\hline \hline Name & \multicolumn{1}{c}{$l$} & \multicolumn{1}{c}{$b$} & \multicolumn{1}{c}{ Alt. Name } \\
\hline F00 & $-0.93^{\circ}$ & $-7.70^{\circ}$ & OGLE-IV-BLG547 \\
F01 & $8.81^{\circ}$ & $-3.64^{\circ}$ & OGLE-IV-BLG527 \\
F02 & $-2.75^{\circ}$ & $-3.32^{\circ}$ & OGLE-2017-BLG-0019 \\
F03 & $3.51^{\circ}$ & $-3.17^{\circ}$ & MOA-II-gb14 \\
F04 & $9.62^{\circ}$ & $-2.93^{\circ}$ & MOA-II-gb21 \\
F05 & $1.83^{\circ}$ & $-2.52^{\circ}$ & OGLE-2015-BLG-0029 \\
F06 & $0.65^{\circ}$ & $-1.86^{\circ}$ & MOA-II-gb5 \\
F07 & $-7.91^{\circ}$ & $-1.85^{\circ}$ & OGLE-IV-BLG672 \\
F08 & $1.25^{\circ}$ & $-1.38^{\circ}$ & OGLE-2014-BLG-0613 \\
F09 & $1.00^{\circ}$ & $-1.03^{\circ}$ & OGLE-IV-BLG500 \\
F10 & $-3.61^{\circ}$ & $1.86^{\circ}$ & OGLE-2015-BLG-0211 \\
F11 & $0.33^{\circ}$ & $2.82^{\circ}$ & OGLE-IV-BLG611 \\
F12 & $7.81^{\circ}$ & $4.81^{\circ}$ & OGLE-IV-BLG629 \\
F13 & $-4.21^{\circ}$ & $4.96^{\circ}$ & OGLE-IV-BLG617 \\
\hline
\end{tabular}

Note - Fields labeled with ${ }^{a}$ are centered on individual microlensing events from the OGLE EWS.

location, and events are detected at $I \lesssim 22$ and the median seeing ranges from 1.25" to 1.35" (Udalski et al. 2015). OGLE also posts alerts about phometric microlensing events through the Early Warning System (EWS). The OGLE EWS reduces photometry in real time, providing information about potential microlensing candidates. With the advent of OGLE-IV, roughly 2000 microlensing events are published each season on the EWS website. ${ }^{5}$

We compare PopSyCLE simulation results to three different papers that use data gathered from these two surveys: Sumi \& Penny (2016), hereafter SP16, which used MOA-II data, and Mróz et al. (2019) and Mróz et al. (2017), hereafter M19 and M17, which used OGLE-IV data. Note that SP16 is an updated version of Sumi et al. (2013) with corrected event rates, due to a correction in the stellar number count. Observational surveys must correct for the ability to detect a given microlensing event. This is quantified by the efficiency correction $\varepsilon$ which is a function of a multitude of parameters, such as the Einstein crossing time, source flux fraction, unlensed source magnitude, survey duration and cadence, and stellar confusion. These three papers calculate efficiency corrections to convert the directly observed distribution of microlensing events to efficiency corrected event rates and $t_{E}$ distributions; we compare

\footnotetext{
${ }^{5}$ http://ogle.astrouw.edu.pl/ogle4/ews/ews.html
}

our PopSyCLE simulations to these efficiency corrected quantities.

We also run PopSyCLE simulations to explore the possibilities of future microlensing surveys. The Wide Field Infrared Survey Telescope (WFIRST) is a 2.4 meter space telescope scheduled to launch in 2025. As part of the program, a microlensing survey searching for exoplanets will observe $1.97 \mathrm{deg}^{2}$ of the Galactic bulge down to $H<26$ every 15 minutes over 6 observing seasons, where each season is 72 days long (Penny et al. 2019, hereafter P19). However, the exact way in which the 6 seasons will be distributed across the survey lifetime of 4.5 - 5 years is not yet determined.

PopSyCLE simulations are run in two parts. We define a field as the projected area on the sky over which PopSyCLE is run, and a simulation is defined to be the particular parameters used and observational criteria imposed on the PopSyCLE run for some field. Table 2 lists the parameters common to all simulations presented in this paper. In summary, for the population synthesis portion of the simulation, we assume that NSs and BHs receive birth kicks of $350 \mathrm{~km} / \mathrm{s}$ and $100 \mathrm{~km} / \mathrm{s}$ in a random direction, respectively. Microlensing events with an impact parameter $u_{0}<2$ are then selected from a $0.34 \mathrm{deg}^{2}$ area survey of 1000 days in length with observations made every 10 days. Table 3 contains a list of all fields run in all simulations. These fields were selected from the OGLE and MOA surveys' Galactic bulge fields, and picked to sample a variety of latitude and longitudes. For WFIRST, only three of these fields are utilized (F06, F08, F09) from the PopSyCLE simulations, as they are representative samples of the WFIRST Cycle 7 design field of view (see Figure 7 of P19).

Note that a survey duration of 1000 continuous days is used in the simulations (Table 2), which does not match those of any of the surveys described above. Survey duration and structure have an effect on the types of events detectable. For example, having gaps between observing seasons can cause a lack of data from one side of the lightcurve, making it difficult to fit for event parameters. Additionally, some events will be undetectable, simply because they are too long to be observed in a particular observing window, or fall entirely within an observational gap. We assume that for published event rates and $t_{E}$ distributions (e.g. SP16; M17; M19) efficiency corrections take these effects into account. However, this statement does not apply to results from the OGLE EWS, as it is only used for alerting events in real time and does not have any type of correction. Similarly for WFIRST, when trying to determine how many BHs are detectable over the duration of the survey, we assume no efficiency correction. The effect that the sea- 
Table 4. Simulation Parameters

\begin{tabular}{|c|c|c|c|c|c|}
\hline \multirow[b]{2}{*}{ Parameter/Criteria } & \multicolumn{5}{|c|}{ Simulation Name } \\
\hline & Mock Sumi & Mock EWS & Mock Mróz19 & Mock Mróz17 & Mock WFIRST \\
\hline Filter & $\mathrm{I}$ & I & $\mathrm{I}$ & I & $\mathrm{H}$ \\
\hline Seeing disk radius, $\theta_{\text {blend }}$ & $-{ }^{a}$ & $0.65^{\prime \prime}$ & $-{ }^{b}$ & $0.65^{\prime \prime}$ & $0.09^{\prime \prime}$ \\
\hline Minimum source magnitude, $m_{S}$ [mag] & $\leq 20$ & - & $\leq 21$ & $\leq 22$ & - \\
\hline Minimum baseline magnitude, $m_{\text {base }}[\mathrm{mag}]$ & - & $\leq 21$ & - & - & $\leq 26$ \\
\hline Maximum impact parameter, $u_{0}$ & $\leq 1$ & $\leq 2$ & $\leq 1$ & $\leq 1$ & $\leq 2$ \\
\hline Removal of highly-blended events, $b_{S F F, m}$ & - & - & - & $\geq 0.1$ & - \\
\hline Einstein crossing time range, $t_{E}[$ days $]$ & {$[0.3,200]$} & - & {$[0.5,300]$} & {$[0.1,316]$} & - \\
\hline Removal of low-amplitude events, $\Delta m$ [mag] & - & $\geq 0.1$ & - & - & $\geq 0.1$ \\
\hline
\end{tabular}

Note - The selection parameters for Mock Sumi, Mróz19, and Mróz17 come from SP16; M19; M17 respectively. For Mock EWS, we choose values based on numbers reported on the OGLE EWS website for the 2016-2018 seasons (described in more detail in $\S 7.2 .1$ ). For Mock WFIRST, the minimum baseline magnitude comes from P19, and the remaining parameter cuts are based on Mock EWS.

${ }^{a}$ There is no cut involving a parameter dependent on $\theta_{b l e n d}$ (such as $b_{S F F}$ or $\Delta m$ ) in the Mock Sumi simulation; however, the median seeing at the MOA site is approximately $2.0^{\prime \prime}$ (SP16), which would roughly correspond to $\theta_{b l e n d}=1.0^{\prime \prime}$.

${ }^{b}$ Similarly to ${ }^{a}$, there is no cut involving a parameter dependent on $\theta_{b l e n d}$; however, the seeing is the same as for M17.

son spacing will have on the number of detectable $\mathrm{BH}$ events is not so obvious, as the exact details of the season distribution have not been set. The effect this has on our estimate of the number of $\mathrm{BH}$ masses that WFIRST can measure will be discussed further in $\$ 7.5$.

Five different simulations are run, named Mock Sumi, Mock Mróz19, Mock Mróz17, Mock EWS, and Mock WFIRST. The first three attempt to mimic the microlensing event selection criteria of SP16, M19, and M17 respectively, after taking into account detection efficiency. These criteria are selected to match the particular magnitude limit, impact parameter, and $t_{E}$ range of the survey's efficiency correction. Mock EWS is designed to mimic the detection capabilities of the OGLE EWS, while Mock WFIRST is designed to mimic WFIRST's microlensing survey based on "reasonable" criteria expected for the mission.

Table 4 lists the corresponding selection criteria for these five simulations.

\section{POPSYCLE COMPARISON}

\subsection{Number of black holes and neutron stars in the Milky Way}

We first use our population synthesis model to calculate the number of BHs and NSs in the Milky Way. First, Galaxia generates a random fraction of the entire Milky Way. We then perform a simplified version of the compact object population synthesis described in $\S 3$ by ignoring the spatial distribution and kinematics of the compact objects, instead only returning the masses of the compact objects. We then scale the number of compact objects in accordance to the fraction of stars generated to obtain the actual number.

With this method, we estimate that there are $2.2 \times 10^{8}$ black holes and $4.4 \times 10^{8}$ neutron stars in the entire Milky Way. Our findings compare well with previous theoretical estimates that predict around $10^{8}$ to $10^{9}$ stellar-mass black holes in the Milky Way, with a similar estimate for neutron stars, using methods based on the number of microlensing events toward the Galactic Bulge (Agol \& Kamionkowski 2002) and supernova explosion and Galactic chemical evolution models (Timmes et al. 1996).

Of the $2.2 \times 10^{8}$ black holes produced by PopSyCLE, $8.5 \times 10^{7}$ have masses greater than $10 M_{\odot}$. This is comparable the findings of Elbert et al. (2018), where they estimate there should be around $10^{8}$ black holes with $M>10 M_{\odot}$ in a galaxy with a stellar mass equal to that of the Milky Way $\left(M_{*, M W} \approx 6 \times 10^{10} M_{\odot}\right)$. Note that the models of Elbert et al. (2018) include metallicity and BH-BH mergers, which PopSyCLE currently does not include. The mass distribution of BHs is shown in Figure 7.1 and a discussion of the distribution is presented in $\S 7.1$.

\subsection{Microlensing event rate}

We next compare stellar densities, event rates, and Einstein crossing times from PopSyCLE simulations with 
Table 5. Comparing PopSyCLE to surveys

\begin{tabular}{lcccccc}
\hline \hline \multirow{2}{*}{ Field } & \multicolumn{2}{c}{$n_{s}\left(10^{6} / \mathrm{deg}^{2}\right)$} & \multicolumn{2}{c}{$\Gamma\left(10^{-6} / \mathrm{star} / \mathrm{yr}\right)$} & \multicolumn{2}{c}{$\left\langle t_{E}\right\rangle$ (days) } \\
& Obs. & Mock & Obs. & Mock & Obs. & Mock \\
\hline $\mathrm{F} 00^{M 19}$ & 2.62 & 2.52 & $1.3 \pm 0.8$ & 5.97 & 32.6 & 20.4 \\
$\mathrm{~F} 01^{M 19}$ & 4.54 & 2.92 & $5.5 \pm 0.9$ & 8.09 & 39.5 & 25.0 \\
$\mathrm{~F} 03^{S}$ & 3.64 & 3.97 & $14.0_{-2.4}^{+2.9}$ & 22.74 & 25.5 & 18.5 \\
$\mathrm{~F} 04^{S}$ & 1.11 & 1.12 & $3.5_{-1.7}^{+2.6}$ & 7.66 & 17.0 & 28.5 \\
$\mathrm{~F} 06^{S}$ & 2.80 & 5.77 & $36.6_{-4.4}^{+4.9}$ & 50.67 & 17.4 & 18.4 \\
$\mathrm{~F} 07^{M 19}$ & 1.75 & 2.09 & $5.6 \pm 1.4$ & 4.11 & 51.8 & 39.5 \\
$\mathrm{~F} 09^{M 19}$ & 4.84 & 4.75 & $23.9 \pm 2.0$ & 43.69 & 18.8 & 17.4 \\
$\mathrm{~F} 11^{M 19}$ & 4.95 & 5.7 & $16.2 \pm 1.3$ & 32.2 & 21.8 & 17.0 \\
$\mathrm{~F} 12^{M 19}$ & 3.26 & 2.07 & $3.4 \pm 1.1$ & 7.25 & 36.7 & 16.7 \\
$\mathrm{~F} 13^{M 19}$ & 4.51 & 3.44 & $5.2 \pm 1.1$ & 11.25 & 30.8 & 21.9 \\
\hline
\end{tabular}

Note-Fields with ${ }^{M 19}$ indicate the observed values come from M19, while those with ${ }^{S}$ are from SP16.

those from M19 and SP16. A summary is presented in Table 5.

For each field listed in Table 3, we apply the observational cuts from Tables 2 and 4 to generate a final list of detectable events as described in $\S 5$. In order to convert to an event rate in units of [events $\operatorname{star}^{-1}$ year $^{-1}$ ], we also calculate the total number of detectable stars within the magnitude limits for the corresponding survey. The microlensing event rate, $\Gamma$, varies between different observational surveys and different fields. The rates for Mock Sumi and Mock Mróz19 are presented in Table 6.

The event rates from SP16 for fields F03, F04, and F06 (corresponding to MOA-II-gb14, gb21, and gb5) are $14.0,3.5$, and 36.6 events star $^{-1}$ year $^{-1}$, respectively. For those same fields, the Mock Sumi PopSyCLE simulation gives event rates of 22.74, 7.66, and 50.67 events $\operatorname{star}^{-1}$ year ${ }^{-1}$. The event rates from PopSyCLE are roughly between 1.4 to 2.2 times higher than the ones reported in SP16.

The event rates from M19 for fields F00, F01, F07, F09, F11, F12, and F13 (corresponding to OGLE-IVBLG547, 527, 672, 500, 611, 629, and 617) are 1.3, $5.5,5.6,23.9,16.2,3.4$, and 5.2 events star $^{-1}$ year ${ }^{-1}$, respectively. For those same fields, the Mock Mróz19 PopSyCLE simulation gives event rates of 5.97, 8.09, 4.11, $43.69,32.2,7.25,11.25$ events star $^{-1}$ year ${ }^{-1}$. The event rates from PopSyCLE are generally a factor of 2 times higher than the ones reported in M19, although for one field it is nearly 5 times higher and in one field it is only 0.7 of the observed rate.

Our event rate estimates from PopSyCLE use a total number of stars that is $100 \%$ complete down to the
Table 6. Mock Simulations

\begin{tabular}{|c|c|c|c|c|c|}
\hline Field & Sim & $\begin{array}{l}n_{s}\left(10^{6}\right) \\
\left(\operatorname{deg}^{-2}\right)\end{array}$ & $\begin{array}{c}\Gamma\left(10^{-6}\right) \\
\left(\operatorname{star}^{-1} \mathrm{yr}^{-1}\right)\end{array}$ & $\begin{array}{c}\left\langle t_{E}\right\rangle \\
\text { (days) }\end{array}$ & $\begin{array}{r}\operatorname{Med}\left(t_{E}\right) \\
\text { (days) }\end{array}$ \\
\hline \multirow[t]{2}{*}{ F00 } & $\mathrm{S}$ & 1.46 & 5.87 & 17.3 & 11.2 \\
\hline & M19 & 2.52 & 5.97 & 20.4 & 14.4 \\
\hline \multirow[t]{2}{*}{ F01 } & $\mathrm{S}$ & 1.52 & 6.37 & 25.1 & 14.8 \\
\hline & M19 & 2.92 & 8.09 & 25.0 & 15.5 \\
\hline \multirow[t]{2}{*}{ F02 } & $\mathrm{S}$ & 2.70 & 19.09 & 20.2 & 14.2 \\
\hline & M19 & 6.11 & 25.66 & 19.5 & 13.9 \\
\hline \multirow[t]{2}{*}{ F03 } & $\mathrm{S}$ & 3.97 & 22.74 & 18.5 & 13.2 \\
\hline & M19 & 7.92 & 30.80 & 19.7 & 14.5 \\
\hline \multirow[t]{2}{*}{ F04 } & $\mathrm{S}$ & 1.12 & 7.66 & 28.5 & 21.6 \\
\hline & M19 & 2.37 & 9.96 & 19.6 & 17.3 \\
\hline \multirow[t]{2}{*}{ F05 } & $\mathrm{S}$ & 5.38 & 38.57 & 17.5 & 13.4 \\
\hline & M19 & 11.05 & 43.65 & 19.5 & 13.9 \\
\hline \multirow[t]{2}{*}{ F06 } & $\mathrm{S}$ & 5.77 & 50.67 & 18.4 & 12.4 \\
\hline & M19 & 12.53 & 59.68 & 18.9 & 12.8 \\
\hline \multirow[t]{2}{*}{ F07 } & $\mathrm{S}$ & 0.99 & 4.33 & 40.9 & 28.1 \\
\hline & M19 & 2.09 & 4.11 & 39.5 & 22.9 \\
\hline \multirow[t]{2}{*}{ F08 } & $\mathrm{S}$ & 2.07 & 33.68 & 20.1 & 16.0 \\
\hline & M19 & 5.02 & 40.04 & 18.1 & 13.1 \\
\hline \multirow[t]{2}{*}{ F09 } & $\mathrm{S}$ & 2.06 & 33.84 & 20.5 & 16.0 \\
\hline & M19 & 4.75 & 43.69 & 17.4 & 12.7 \\
\hline \multirow[t]{2}{*}{ F10 } & $\mathrm{S}$ & 0.33 & 19.69 & 13.4 & 13.0 \\
\hline & M19 & 0.64 & 33.32 & 24.5 & 15.1 \\
\hline \multirow[t]{2}{*}{ F11 } & $\mathrm{S}$ & 2.05 & 21.53 & 16.6 & 10.1 \\
\hline & M19 & 5.70 & 32.20 & 17.0 & 11.4 \\
\hline \multirow[t]{2}{*}{ F12 } & $\mathrm{S}$ & 1.11 & 5.80 & 18.1 & 10.1 \\
\hline & M19 & 2.07 & 7.25 & 16.7 & 9.1 \\
\hline \multirow[t]{2}{*}{ F13 } & $\mathrm{S}$ & 1.68 & 6.40 & 12.3 & 7.9 \\
\hline & M19 & 3.44 & 11.25 & 21.9 & 13.1 \\
\hline
\end{tabular}

Note-For each field simulation $(\mathrm{S}=$ Mock Sumi, M19 = Mock Mróz19) we list the density of stars $n_{s}$ brighter than the survey magnitude limit (no correction for blending or confusion), the event rate $\Gamma$, average Einstein crossing time $\left\langle t_{E}\right\rangle$, and the median Einstein crossing time $\operatorname{Med}\left(t_{E}\right)$.

selected magnitude, as we have not imposed any confusion due to the finite beam size of a real telescope. We assume that the reported event rates in Sumi \& Penny (2016); Mróz et al. (2017, 2019) use completenesscorrected stellar number counts.

\subsection{Einstein Crossing Time Distribution}

The average Einstein crossing time $\left\langle t_{E}\right\rangle$ is a commonly reported parameter for microlensing distributions. Most authors refer to both the raw $\left\langle t_{E}\right\rangle$ derived from the uncorrected distribution obtained directly after making all observational cuts, and an efficiency corrected $\left\langle t_{E}\right\rangle_{\varepsilon}$, obtained after correcting for the detection efficiency. In this section, we compare the reported $\left\langle t_{E}\right\rangle_{\varepsilon}$ from SP16 and M19 to those obtained from PopSyCLE simulations. For brevity, whenever the notation $\left\langle t_{E}\right\rangle$ is used, it is understood to be efficiency corrected, unless otherwise stated. 
The $\left\langle t_{E}\right\rangle$ from SP16 for fields F03, F04, and F06 are $25.5,17.0$, and 17.4 days, respectively. For these same fields, the Mock Sumi PopSyCLE simulation gives $\left\langle t_{E}\right\rangle$ of $18.5,28.5,18.4$ days; these values range between factors of 0.7 to 1.7 times the observed timescales.

The $\left\langle t_{E}\right\rangle$ from M19 for fields F00, F01, F07, F09, F11, F12, and F13 are 32.6, 39.5, 51.8, 18.8, 21.8, 36.7, and 30.8 days, respectively. For these same fields, the Mock Mróz19 simulation gives $\left\langle t_{E}\right\rangle$ of 20.4, 25.0, 39.5, 17.4, $17.0,16.7$, and 21.9 days; these values are all shorter than the observed timescales by factors of 0.5 to 0.9 .

Values for $\left\langle t_{E}\right\rangle$ for the events in M17 cannot be calculated as they do not report nor present individual event parameters (only the values binned into the histogram are given). Thus we compare the entire $t_{E}$ distribution to PopSyCLE, and in particular the "peak" of the $t_{E}$ distribution $\left(t_{E, \text { peak }}\right)$, defined as the location of the maximum of the $t_{E}$ distribution when the individual timescales are binned logarithmically. The peak of a logarithmically binned distribution is not the best quantity to compare, and we advocate performing a fit to the $t_{E}$ distribution instead. However, for the moment we use $t_{E, \text { peak }}$ as a proxy for some measure of central tendency (note that $t_{E, \text { peak }}$ does not correspond to the mean, median, nor mode of the $t_{E}$ values).

From the efficiency-corrected $t_{E}$ distributions presented in several papers, $t_{E, \text { peak }}$ is located around 15-20 days (see Figure 13 of Sumi et al. (2013), Figure 17 of Wyrzykowski et al. (2015), and Figure 2 of M17). As can be seen in Figure 6.3, $t_{E, \text { peak }}$ from PopSyCLE falls in the lower end of that range.

\section{RESULTS}

\subsection{Milky Way Present-Day BH Mass Function}

The BH present-day mass function (PDMF) encodes information about the BH IFMR and stellar IMF, and to a lesser degree the star formation history (SFH). It also provides information about $\mathrm{BH}$ binaries and their formation channel(s). With a sufficiently large sample of BH mass measurements from both LIGO (extragalactic) and microlensing (Galactic), the BH PDMF can be measured and the IFMR can be constrained.

We use PopSyCLE to generate the Milky Way BH PDMF which is shown in Figure 7.1. The R18 IFMR clearly shows the "mass gap", as the lowest mass BH is around $5 M_{\odot}$, which is greater than the largest possible NS mass $M_{N S, \max } \sim 2-3 M_{\odot}$ (Özel \& Freire (2016), but also see Margalit \& Metzger (2017) which suggests an upper limit closer to $\sim 2.2 M_{\odot}$ ). As discussed in $\S 2.2 .1$, there are no $\gtrsim 30 M_{\odot}$ black holes as the R18 IFMR assumes only solar metallicity progenitor stars and PopSyCLE currently implements only single BHs.

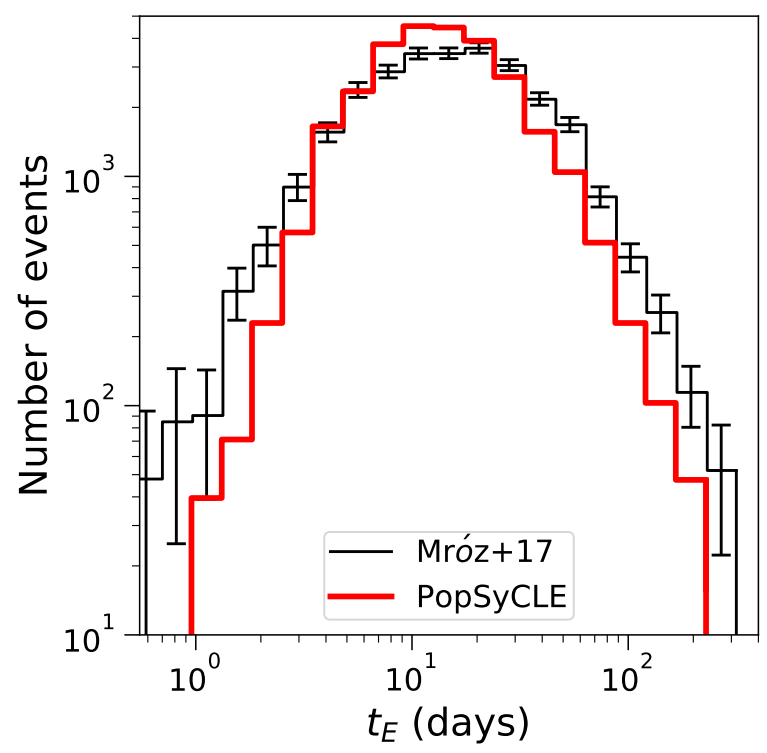

Figure 4. The $t_{E}$ distribution in black comes from Figure 2 of M17. Overplotted in red is the Mock Mróz17 PopSyCLE simulation, scaled such that the number of events are the same.

For the Milky Way, the SFH (according to the Besançon model) does not influence the BH PDMF very much, as most stars are over $10^{9}$ years old. The minimum ZAMS mass for a star to form a black hole is $\sim 15 M_{\odot}$, and the corresponding main-sequence lifetime of such a star is $\sim 10^{7}$ years. Thus, the vast majority of BHs produced when their progenitor star dies will have already been formed.

The R18 IFMR also produces structures such as peaks and gaps in the BH PDMF. The spike around $6 M_{\odot}$ is due to a combination of stars with ZAMS masses between $15-20 M_{\odot}$ and $70-120 M_{\odot}$. Although there are more stars of lower ZAMS masses due to the IMF, only $34 \%$ of stars within $15-20 M_{\odot}$ form BHs, while $60 \%$ of the $>70 M_{\odot}$ stars form BHs. The paucity around $10 M_{\odot}$ is due to the fact that most stars between $25-27 M_{\odot}$ form NS and not BHs. The general decrease in number of $\mathrm{BHs}$ greater than $8 M_{\odot}$ is simply due to the IMF; high mass stars are rarer. These trends are shown visually in Figure 7.1, which combines the IFMR with the IMF and $\mathrm{SFH}$. We re-emphasize that this structure is specific to the R18 IFMR; a different IFMR will produce a different BH PDMF. However, with this assumption, the structure in the BH PDMF may be detectable with a sample of $\sim 100$ BHs. This will become a possiblity with WFIRST, as discussed in $\S 7.5$. 


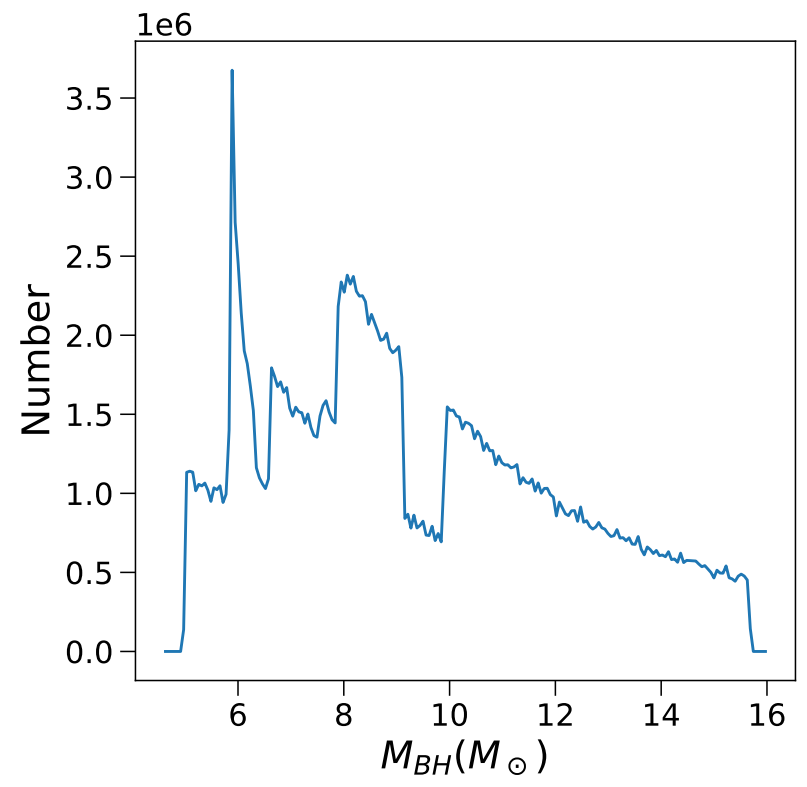

Figure 5. The Milky Way BH present-day mass function. The description of $\mathrm{BH}$ population synthesis used to produce this distribution is described in $\S 6.1$. Note that this is the theoretical underlying distribution for the entire Milky Way, not just those observable via microlensing; no observational constraints or limitations have been taken into account. The BH IFMR comes from R18.

\subsection{Measuring Black Holes Masses with Individual Astrometric Follow-up}

Identifying a $\mathrm{BH}$ with microlensing requires showing that the lens mass exceeds $5 M_{\odot}$ and is not luminous. Since $5 M_{\odot}$ stars are extremely bright, it is relatively easy to rule out massive stellar lenses once a lens mass is in hand. However, as described in $\S 4.2$, photometric microlensing alone is not sufficient to determine the mass of the lens in a microlensing event; astrometry is also needed to break degeneracies between lens mass and source and lens distances. Thus, data from a photometric survey must be combined with astrometric followup of a smaller set of $\mathrm{BH}$ candidates. As an example, we investigate the strategy of $\mathrm{Lu}$ et al. (2016) of selecting astrometric follow-up candidates with long Einstein crossing times $t_{E} \gtrsim 120$ days, high magnification $A_{\text {max }} \gtrsim 10 \equiv u_{0} \lesssim 0.1$, and high source flux fraction $b_{S F F} \approx 1$ from photometric surveys updates such as the OGLE EWS (described in $\S 5$ ).

\subsubsection{Selecting BH Candidates with OGLE}

Out of all the Mock EWS events, $1 \%$ of them have a $\mathrm{BH}$ lens. This is consistent with the fraction of $\mathrm{BH}$ lensing events in the unfiltered PopSyCLE event candidates list as well. While BHs make up $\lesssim 0.1 \%$ of ob-

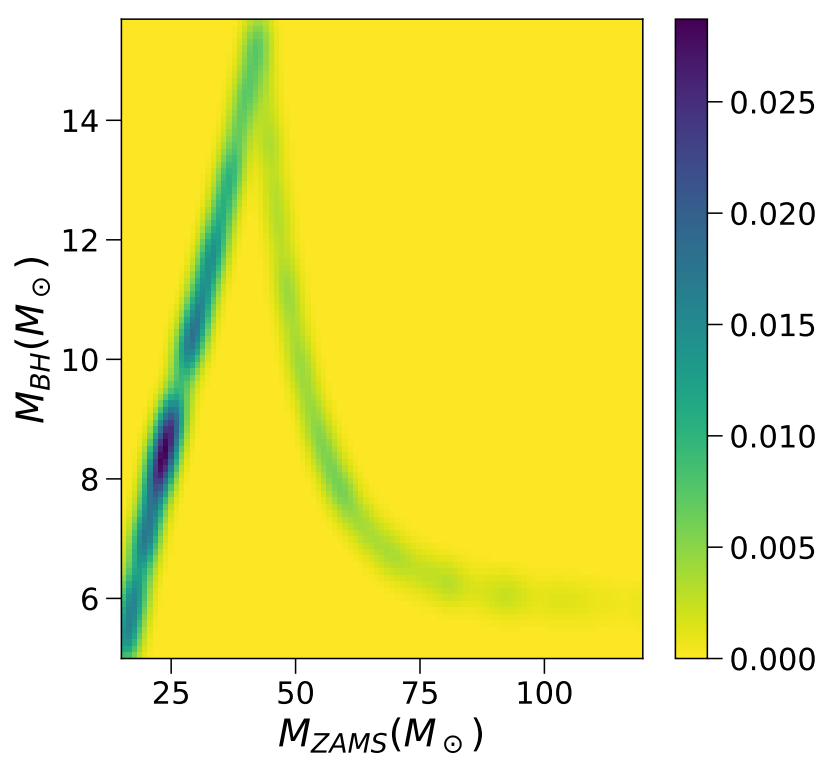

Figure 6. The black hole initial-final mass relation $(\mathrm{BH}$ IFMR), with the colorbar indicating the density of Milky Way stars at that part of the IFMR. For example, there is an overall trend where there are many more lower mass progenitors; this is mainly due to the IMF. However within that overall framework, there is more structure, which comes from the fact that some of the progenitor stars will form NSs and not BHs. The BH IFMR provides a mapping between the ZAMS mass of a star and the mass of the BH it forms, assuming it forms a BH and not a neutron star. It is described by a piecewise function (Equation $\mathrm{C} 1$, with ejection fraction $\left.f_{e j}=0.9\right)$. This value of $f_{e j}$ was selected as it most closely reproduced the observed distribution of black hole masses (R18).

jects in the Milky Way by number, their lensing rate is enhanced by their $\sim 10 \times$ larger Einstein radius. Thus, the observational selection criteria of the OGLE survey does not dramatically change the probability of detecting $\mathrm{BH}$ lensing events. In Figure 7, the various types of lenses contributing to the $t_{E}$ distribution is shown, which is comparable to the efficiency-corrected $t_{E}$ curves produced by surveys. Figure 7.2.1 shows roughly the number of candidates that can be followed up based on EWS photometry per season. Although there usually are nearly 100 events with $t_{E}>120$ days, often only 10 or so of those have sufficiently high SNR data and good coverage of the event, which are necessary later when trying to fit the event and extract the microlensing parameters $t_{E}$ and $\pi_{E}$.

Selecting Mock EWS events with $t_{E}>120$ days increases the probability of the lens being a BH to $40 \%$ (Figure 9). Note that a different Galactic model can increased this fraction by up to a factor of two (see Ap- 

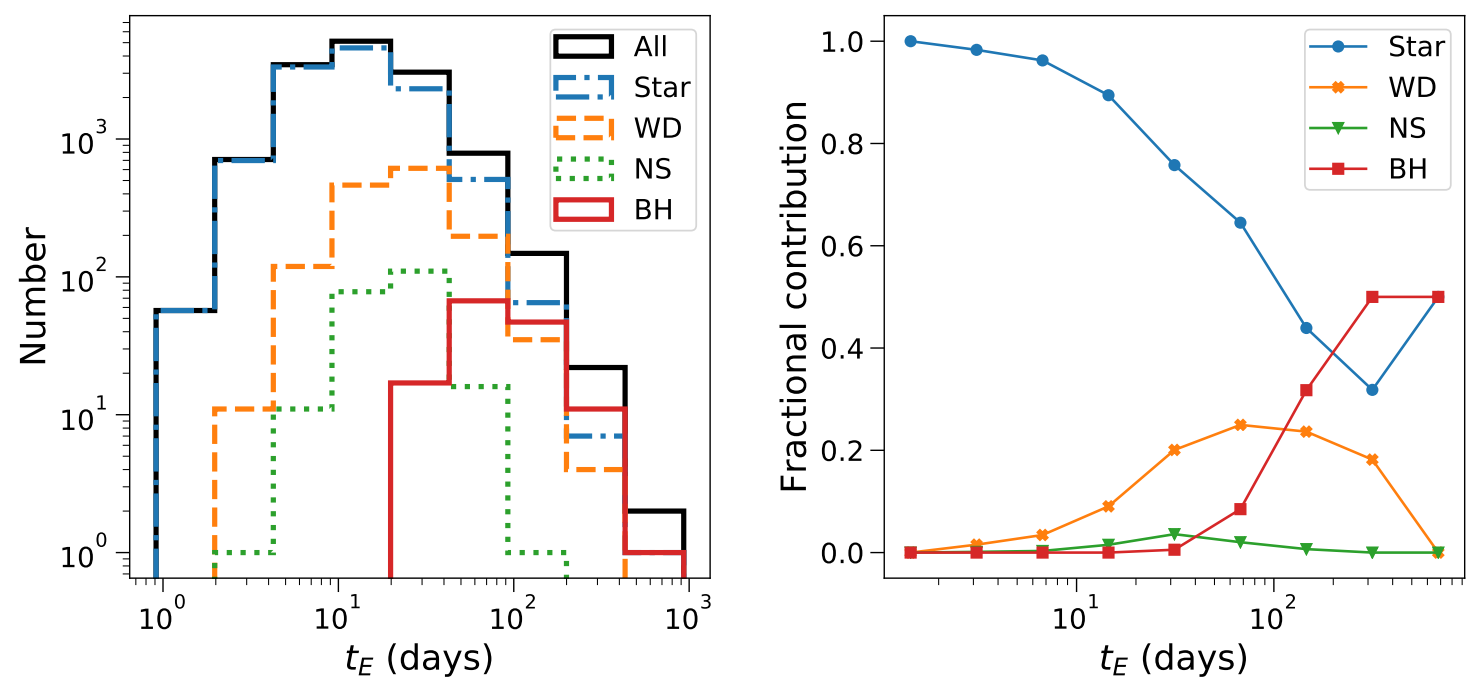

Figure 7. Left: $t_{E}$ distribution from the Mock EWS simulation, showing the different contributions due to different components. Right: The fractional contribution from each type of object type at different $t_{E}$ times. Note that the NS and BH contributions can be shifted to different $t_{E}$ depending on the adopted kick velocities. Also note that many of these events would be difficult to detect as they have parameters with low detection efficiency; however, it serves to illustrate the underlying contributions to the $t_{E}$ distribution.

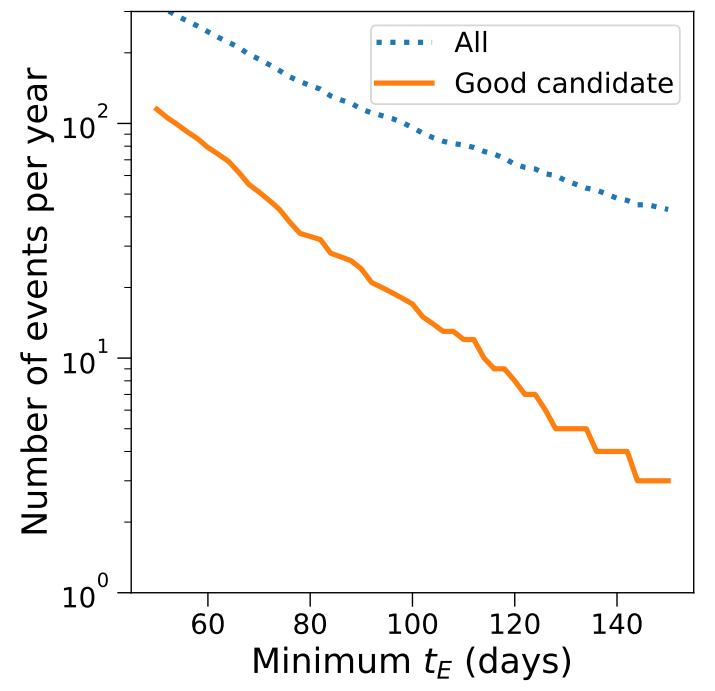

Figure 8. Number of events per season from the OGLE Early Warning System that have $t_{E}$ larger than some minimum value (dotted blue line). However, even events with $t_{E}>120$ days are not necessarily good follow-up candidates, as they may not have sufficient coverage of the photometric peak or insufficient SNR. Thus, good candidates have 1) $\pm 0.5 t_{E}$ coverage of the peak, 2) $\left.I_{\text {base }}<19.5 \mathrm{mag}, 3\right)$ $\Delta I>0.5 \mathrm{mag}$ (orange line). The numbers plotted are the averages over the 2016, 2017, and 2018 seasons reported on the OGLE EWS website.

pendix A). We then explored two additional selection criteria in addition to long events.

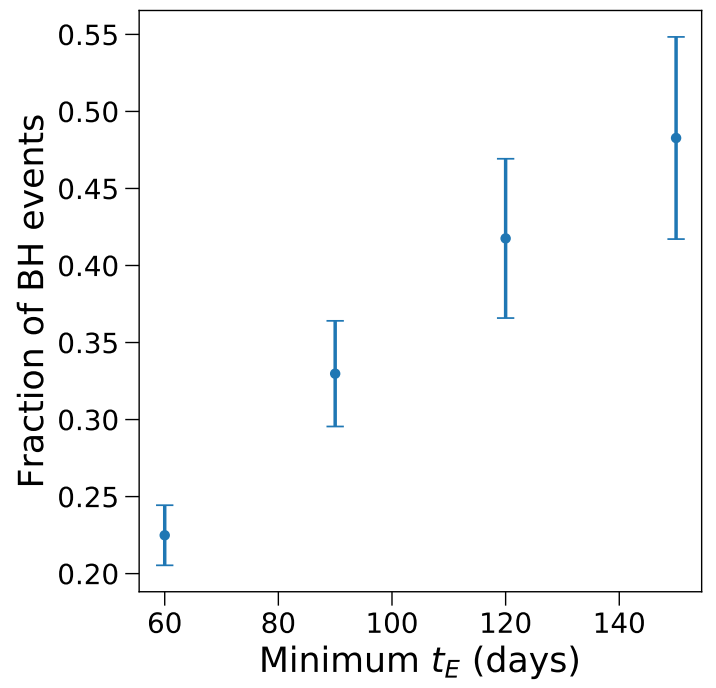

Figure 9. Fraction of long-duration Mock EWS events from PopSyCLE that have BH lenses. $t_{E}>120$ day selections are still recommended for maximizing the probability of finding a BH lens.

First, we tried selecting on events from PopSyCLE simulations with $b_{S F F} \approx 1$. Intuitively, one would expect a source to be less blended with a $\mathrm{BH}$ lens than with a stellar lens, as the BH does not contribute any flux. However, for ground based surveys the seeing disk is so large that many background stars fall within the disk, and whether the lens is luminous or not does not sig- 
nificantly change $b_{S F F}$. In principle, additional highresolution imaging from space or ground-based telescopes with adaptive optics provides a much smaller seeing disk, reducing contamination from neighboring stars. This would then circumvent the aforementioned problem, meaning BH lensing events would truly have a high $b_{S F F}$. However, this strategy fails, as OGLE microlensing events are observationally biased toward brighter sources and the average lens is quite faint $(I \sim 26)$. Thus, the distribution of $b_{S F F}$ for BH and stellar lenses are similar in a sample limited to $\mathrm{I} \lesssim 21$.

Similarly, selecting events with small $u_{0}$ does not increase the probability of finding a BH lens. Although the average $\mathrm{BH}$ is more massive than the average star and thus has a larger $\theta_{E}$, it does not have a correspondingly smaller $u_{0}$, as $u_{0}$ is independent of mass.

\subsubsection{Individual Candidate Follow-up}

As our ultimate goal is to measure the number of $\mathrm{BHs}$ in the Milky Way, we now consider how many BHs can be expected to be detected via astrometric follow-up. We estimate that $\sim 12 \mathrm{BH}$ candidates (corresponding to 5 expected $\mathrm{BH}$ detections) are required to constrain the total number of BHs in the Milky Way to 50 percent, assuming Poisson statistics (Figure 7.2.2). With an astrometric follow-up program, due to practicalities such as limited telescope time on facilities able to perform such measurements, 3-4 candidates can be observed each year and the probability of the candidate being a $\mathrm{BH}$ is $\sim 40 \%$; thus about 1 - 2 BHs per year can be expected to be detected. Over 5 years, this would result in a total of 5 - 10 BHs. However, the BH PDMF would be difficult to constrain based on current sample sizes and sporadic astrometric follow-up, due to the inefficiency of the process. Dedicated astrometric surveys are needed to place useful constraints on the BH PDMF.

\subsection{Confirming BH Lenses with Astrometry and Microlensing Parallax}

$\mathrm{BH}$ candidates identified from photometric microlensing surveys can be followed up with high-precision astrometric measurements in order to measure the astrometric microlensing shift, $\delta_{c, \max }$. When combined with $t_{E}, \pi_{E}$, and $u_{0}$ from the photometric light-curve, the astrometric shift yields a constraint on the lens mass. In Figures 7.3 and 7.3, microlensing events are plotted in $\delta_{c, \max }-\pi_{E}$ space. In Figures 7.3 and 7.3 the microlensing events are plotted in $\pi_{E}-t_{E}$ and $\delta_{c, \max }-t_{E}$ space, respectively. We note that blending due to the lens (if it is luminous) is incorporated into the astrometric shift calculations, assuming a $\sim 100$ mas aperture size that is roughly equivalent to infrared imaging with JWST or with an 8-10 meter telescope and an adaptive

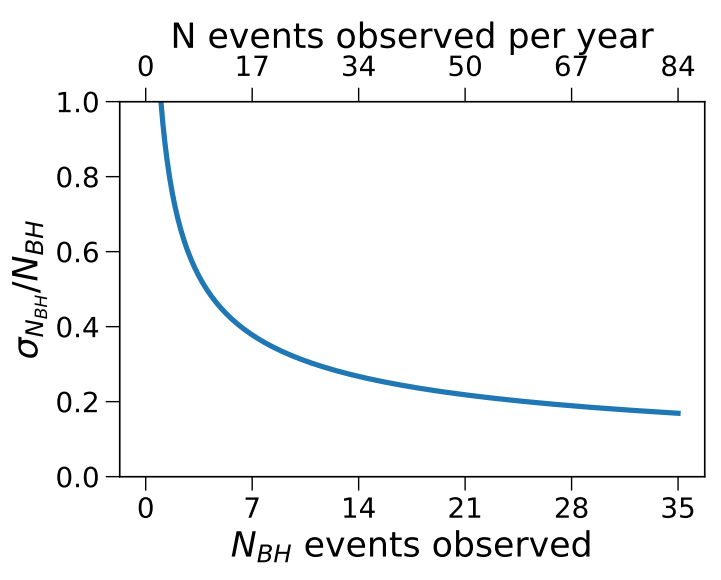

Figure 10. Number of $\mathrm{BH}$ candidates needed to constrain the total number of BHs in the Milky Way to some uncertainty (top horizontal axis), assuming Poisson uncertainty. The number of actual BHs resulting from the sample of candidates is the bottom horizontal axis. This assumes we have selected candidates from long-duration $\left(t_{E}>120\right.$ days) OGLE EWS following the strategy outlined in $\S 7.2 .1$.

optics system. BHs occupy a very particular region in $\delta_{c, \max }-\pi_{E}$ space (large $\delta_{c, \max }$ and small $\pi_{E}$ ) as they are massive. Although both a massive luminous stellar lens and equally massive black hole will have equal Einstein radii, all else equal, the astrometric shift will be larger for the $\mathrm{BH}$, as it do not blend with the source images (see Equation 10 vs. Equation 11).

The very sharp delineation between the stars, WDs, $\mathrm{NSs}$, and BHs in Figure 7.3 can be simply explained. Consider a lens of mass $M$. Using Equations 3, 14, 15, and the definition of $\kappa$ given in $\S 4.2$, the microlens parallax will simply be

$$
\pi_{E}=\sqrt{\frac{\pi_{r e l}}{\kappa M}} .
$$

Assuming an impact parameter of $u_{0} \leq \sqrt{2}$, the maximum astrometric shift, assuming no blending, can be then written

$$
\delta_{c, \max }=\sqrt{\frac{\kappa M \pi_{r e l}}{8}}
$$

by combining Equations 3, 10, and 15. Thus, for a given mass $M$, both $\pi_{E}$ and $\delta_{c, \text { max }}$ scale as $\sqrt{\pi_{r e l}}$. Hence, when plotting $\delta_{c, \max }$ against $\pi_{E}$ for a given mass lens, the slope is 1 . Since PopSyCLE currently assumes that all NSs are of the same mass, they lie artificially on a straight line. Similarly, as there exists a minimum mass for BHs, WDs, and stars, there is a hard edge on the right diagonal side of those populations. There is some downward scatter in $\delta_{c, \max }$ due to some events with $\sqrt{2}<u_{0}<2$, as the maximum astrometric shift 

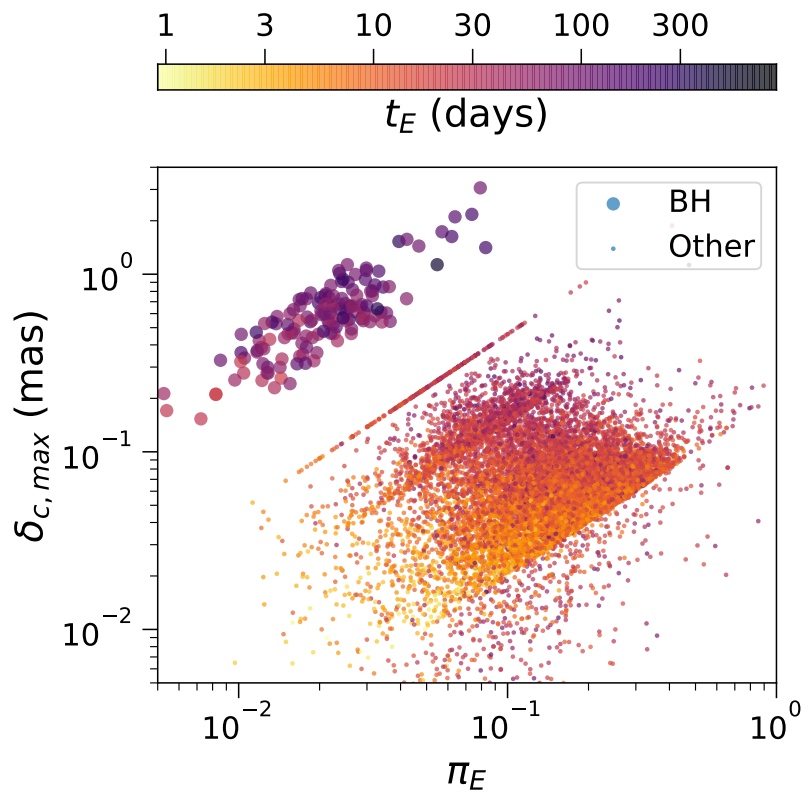

Figure 11. The maximum astrometric shift $\delta_{c, \max }$ vs. the microlensing parallax $\pi_{E}$, with the color of the point indicating the Einstein crossing time $t_{E}$ of the event. We include blending between the lens and source when calculating $\delta_{c, \max }$. The BHs (large points) are easily seperable in this space. The points correspond to microlensing events from the Mock EWS simulation.

is less than what would be given in Equation 18. Additionally when blending is included, $\delta_{c, \max }$ scatters lower for some stars, depending on the lens luminosity. The larger scatter from the slope of 1 in the $\mathrm{BH}, \mathrm{WD}$, and stellar populations are simply due to the fact that there are a range of masses.

Equations 17 and 18 can also be used to understand the $t_{E}$ gradient stretching from the bottom right (large $\pi_{E}$, small $\delta_{c, \max }$ ) to the top left (small $\pi_{E}$, large $\delta_{c, \max }$ ) shown in Figure 7.3. Since $t_{E}$ scales with the square root of the lens mass, heavier lenses will have smaller $\pi_{E}$, larger $\delta_{c, \max }$, and longer $t_{E}$, on average. Scatter in this relation is due to the fact that $t_{E}$ is also degenerate with the relative source-lens proper motion $\mu_{\text {rel }}$.

\subsection{Statistical Samples of Black Holes from Photometry Alone}

The microlensing parallax $\pi_{E}$ combined with a measurement of the Einstein crossing time $t_{E}$ appears to be a powerful means of picking out BHs (Figure 7.3). Unfortunately, $\pi_{E}$ can only be observed significantly after the photometric peak, and thus cannot be used as a BH-candidate selection criteria for astrometric followup. However, the combination of $\pi_{E}$ and $t_{E}$ can still be used to obtain a sample of microlensing events with a very high fraction of $\mathrm{BH}$ lenses, as compared to looking

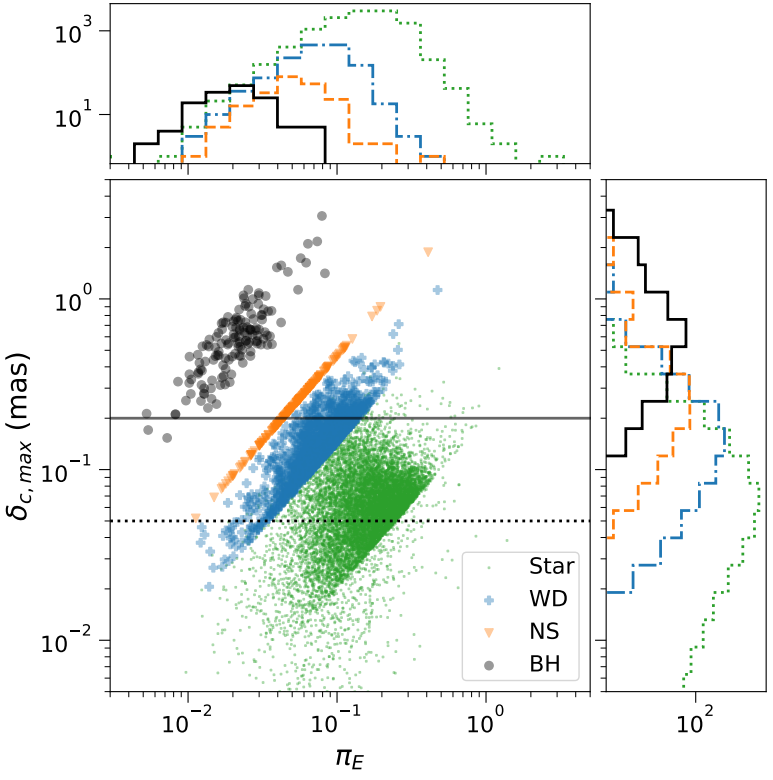

Figure 12. The maximum astrometric shift $\delta_{c, \max }$ vs. the microlensing parallax $\pi_{E}$ for different lens types. Blending between the lens and source is included when calculating $\delta_{c, \max }$. The solid line denotes the current astrometric precision ( $~ 0.2$ mas, using the Keck laser guide star adaptive optics system). The dotted line denotes anticipated astrometric precision achievable in the next decade $(\sim 0.05$ mas, using WFIRST or the Thirty Meter Telescope). The points correspond to microlensing events in the Mock EWS simulation.

at $t_{E}$ alone. By selecting events with both $t_{E}>120$ days and a microlensing parallax of $\pi_{E}<0.08$, the detection rate of $\mathrm{BHs}$ is $\sim 85 \%$; by using an even lower value of $\pi_{E}$, the minimum value of $t_{E}$ could be shifted to lower values and still preserve the high fraction of BHs. This has the additional advantage that $t_{E}$ and $\pi_{E}$ are quantities that can be fit from photometry alone. Thus, with a set of photometric lightcurves, events can be sorted into $\mathrm{BH}$ and non-BH lenses with high statistical confidence.

This method is only useful if the $\pi_{E}$ distribution does not vary dramatically as the Galactic model changes. It is not entirely clear how distinct this separation will be if different assumptions are made about the underlying distributions of $M, d_{L}, d_{S}$, and $\mu_{r e l}$. We evaluate the impact on the $\pi_{E}-t_{E}$ space by using the scaling relations,

$$
\begin{aligned}
t_{E} & \propto \frac{\sqrt{\pi_{r e l} M}}{\mu_{r e l}} \\
\pi_{E} & \propto \sqrt{\frac{\pi_{r e l}}{M}} .
\end{aligned}
$$

as illustrated in the arrows on Figure 7.3. 


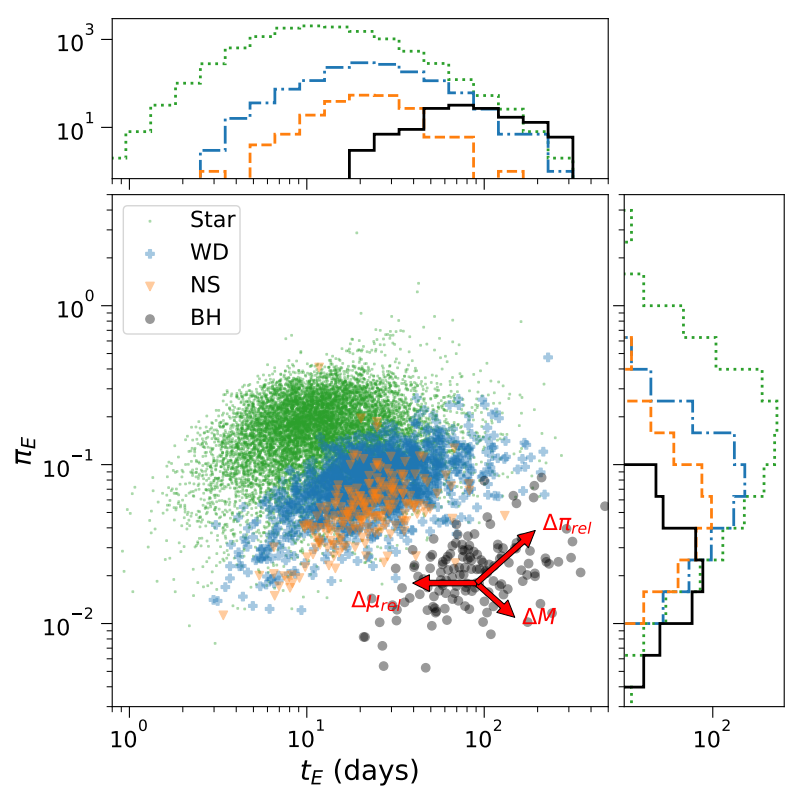

Figure 13. The microlensing parallax $\pi_{E}$ vs. the Einstein crossing time $t_{E}$. The points correspond to microlensing events in the Mock EWS simulation. The red arrows correspond to the effects of changing $\pi_{r e l}, \mu_{r e l}$, and $M$. Consider the fiducial parameters $d_{L}=6.89 \mathrm{kpc}, d_{S}=8.62 \mathrm{kpc}$, $\mu_{\text {rel }}=6.55 \mathrm{mas} / \mathrm{yr}$, and $M=11.12 M_{\odot}$; this corresponds to $t_{E}=90.50$ days and $\pi_{E}=0.018$. If the lens mass $M$ is increased to $30 M_{\odot}$ and all other parameters are held fixed, then $t_{E}=148.64$ days and $\pi_{E}=0.011$. If the relative proper motion $\mu_{r e l}$ is increased to 15 mas/yr and all other parameters are held fixed, then $t_{E}=39.53$ days and $\pi_{E}$ does not change. If the relative parallax $\pi_{r e l}$ is increased from 0.029 mas to 0.13 mas by changing $d_{L}$ to $4 \mathrm{kpc}$ and all other parameters are held fixed, then $t_{E}=193.93$ days and $\pi_{E}=0.039$.

Of all the populations in PopSyCLE, the most uncertainty lies in the $\mathrm{BH}$ spatial positions, velocities, and masses. We consider the effects of changing these parameters. The following trends are illustrated with specific values in Figure 7.3.

- Our BH mass distribution ranges from $5 M_{\odot}$ to 16 $M_{\odot}$; by including more high-mass BHs such as the ones discovered by LIGO into the distribution, the $\mathrm{BH}$ population would shift toward longer $t_{E}$ and smaller $\pi_{E}$.

- The kick velocities of BHs at birth, if there are any, is unknown. Changes in kick velocity are manifested in changes in $\mu_{r e l}$. If $\mu_{r e l}$ increases (holding $d_{L}$ and $d_{S}$ fixed) $t_{E}$ becomes shorter; if $\mu_{r e l}$ increases, $t_{E}$ becomes shorter.

- The spatial distribution of BHs is also not known. Two extremes could be considered: a centrally concentrated population of BHs in the bulge, or a

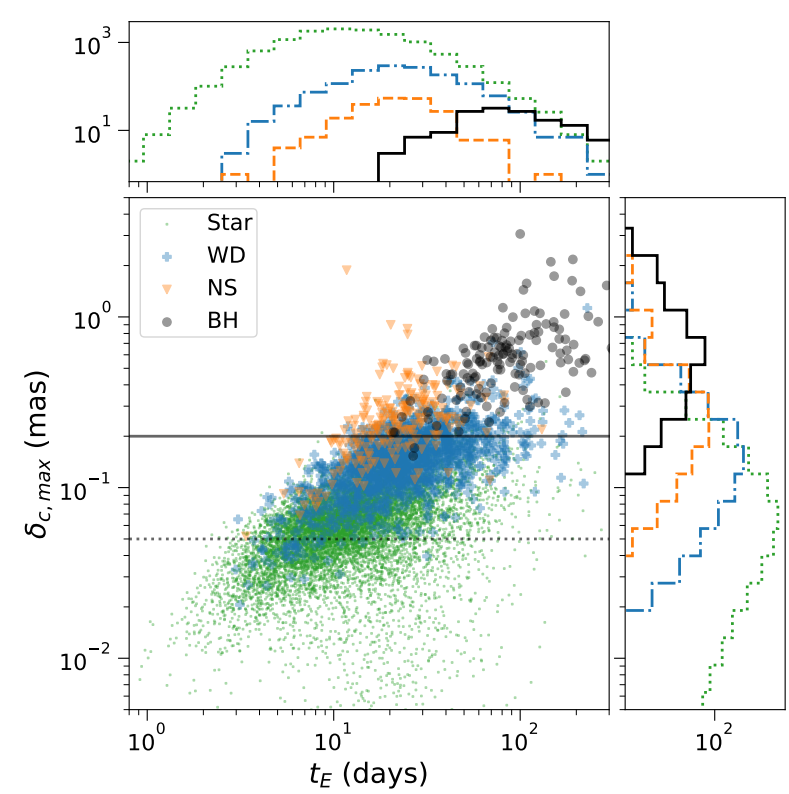

Figure 14. The maximum astrometric shift $\delta_{c, \max }$ vs. the Einstein crossing time $t_{E}$. We assume blending between the lens and source when calculating $\delta_{c, \max }$. The solid line denotes the achievable astrometric precision of $\sim 0.2$ mas using the Keck laser guide star adaptive optics system ( $\mathrm{Lu}$ et al. 2016). The dotted line denotes anticipated astrometric precision achievable in the next decade (e.g. $\sim 0.05$ mas, using WFIRST or the Thirty Meter Telescope). The points correspond to microlensing events in the Mock EWS simulation.

distribution spread throughout the stellar or dark matter halo. Bulge lenses have smaller $\pi_{r e l}$, while disk lenses have a larger $\pi_{r e l}$. Thus, having only $\mathrm{BH}$ bulge lenses would cause the average $\pi_{r e l}$ to increase, causing both $\pi_{E}$ and $t_{E}$ to increase. On the other hand, with a non-centrally concentrated distribution of BHs, this would cause more lenses to fall closer to Earth, meaning the average $d_{L}$ and thus $\pi_{r e l}$ would decrease, causing $\pi_{E}$ and $t_{E}$ to decrease.

Although this method of selecting BH lenses does not allow for direct confirmation nor mass measurement, it does allow for a statistical analysis of BH lensing events. For example, the number of BHs in the Milky Way could be constrained; their masses could also be estimated by invoking some type of Galactic model, as is commonly done. It's strength is that no additional data is necessary and the improved $\mathrm{BH}$ selection process can still lead to improved physical constraints.

The challenge associated with this method is constraining $\pi_{E}$ accurately down to the level which is necessary for this measurement. However, with high cadence observations and high photometric precision this is possible. 


\subsection{BH Hunting with WFIRST}

Lastly, we consider future microlensing surveys and the prospects they hold for measuring $\mathrm{BH}$ masses. As described in $\S 5$, the WFIRST mission includes a microlensing survey designed for exoplanet detections. Some primary differences between the OGLE and WFIRST surveys will be their filters, sensitivity, and resolution (seeing limited $I \lesssim 22$ vs. diffraction limited $H \lesssim 26$, respectively). An additional important difference is that with WFIRST, the astrometry will be obtained at the same time as the photometry. Thus, it is not necessary to do individual follow-up of candidate $\mathrm{BH}$ events; all the information will be contained within the WFIRST survey data. Simultaneous astrometry also has the advantage that it is possible to go "back in time" to look at astrometric data from before the photometric peak (i.e. before the microlensing event is recognized photometrically).

P19 presents thorough simulations and a detailed analysis to calculate the expected yield of bound planet detections with WFIRST microlensing. We present here a complementary analysis with PopSyCLE to determine the number of BHs we may expect a WFIRST-like survey to find. Detailed simulations of different season distributions and understanding how the $\mathrm{BH}$ yield changes is beyond the scope of this paper and is left as future work. However our continuous 1000 day survey simulation with Mock WFIRST parameters (Table 4) is a good order-of-magnitude estimation, considering the other uncertainties in the PopSyCLE simulation and the WFIRST survey design itself.

To normalize the Mock WFIRST survey area to that of the actual WFIRST microlensing survey, the number of events is multiplied by the ratio of the areas (a factor of 1.93). Microlensing events from the Mock WFIRST simulation with $\mathrm{BH}$ lenses and an Einstein crossing time of $90<t_{E}<300$ days are defined to have "measureable" BH masses. Note that there will certainly be many $\mathrm{BH}$ microlensing events with $t_{E}$ that fall above or below this range; however, we do not consider those to have measureable masses, for the following reason. As described in $\S 4.2$, in addition to the photometric brightening, it is necessary to measure the astrometric shift and microlensing parallax to constrain the lens mass. The choice for the lower bound on $t_{E}$ takes into the consideration that an event needs to have a somewhat long duration $\left(t_{E} \gtrsim 3\right.$ months) to have a potentially measureable microlensing parallax $\pi_{E}$. The choice for the upper bound on $t_{E}$ takes into consideration that the astrometric signal falls off much more slowly than the photometric signal; a rough guess is that to measure $\delta_{c, \max }$ requires astrometric data for $\sim 5-10 t_{E}$. Note the

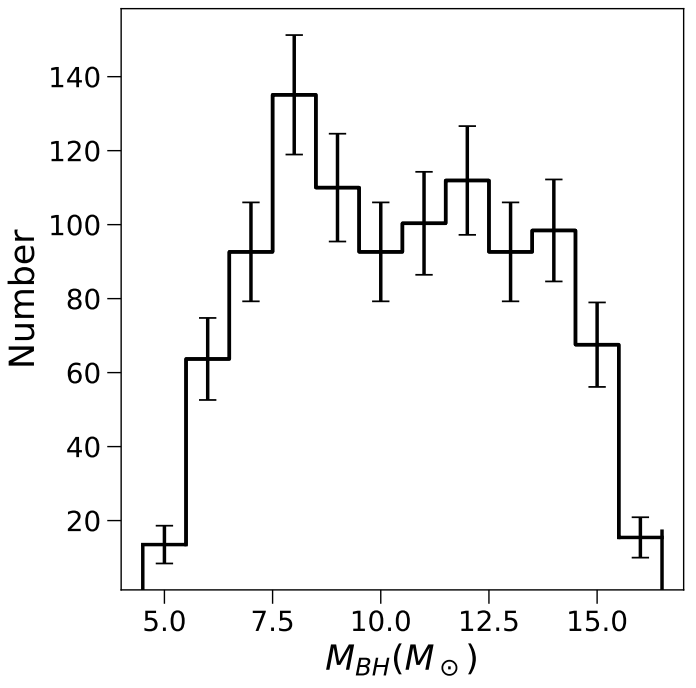

Figure 15. Histogram of the $\mathrm{BH}$ masses that are measurable from the Mock WFIRST BH simulation, scaled to match the area of the actual WFIRST survey. To infer the underlying $\mathrm{BH}$ PDMF, an observational completeness correction will be required in order to account for the astrometric bias towards heavier lenses. PopSyCLE is ideally suited for forward modeling populations, including completeness corrections.

amount of astrometric data required also is dependent on when the astrometric measurements occur. To properly determine this requires detailed simulation (e.g. $\S 8$ of Lu et al. (2016)) and is beyond the scope of this paper.

With this definition, the PopSyCLE Mock WFIRST survey produces $\sim 1000 \mathrm{BH}$ lensing events with measureable masses, nearly 100 times more than with an individual astrometric follow-up program, as estimated in $\$ 7.2 .2$. With this number of mass measurements, we can constrain the present-day BH mass function (Figure 7.5) and the results of supernova simulations.

A major source of uncertainty in this estimate is due to the Galactic model, in both the stellar and compact object components. As described in Appendix A, we consider two different angles $\left(\alpha=11.1^{\circ}\right.$ and $\left.\alpha=28^{\circ}\right)$ for the Galactic bar/bulge; this modification significantly changes the number of stars along a given line of sight. The more tilted bar with $\alpha=11.1^{\circ}$ produces $4-5$ times less microlensing event candidates than the less tilted bar with $\alpha=28^{\circ}$. By applying the Mock WFIRST criterion to the six fields in Table 7, the number of events with measureable $\mathrm{BH}$ masses is decreased by a factor of about 4 . To take into account these uncertainties, we estimate the number of $\mathrm{BHs}$ with measureable masses is $\mathcal{O}(100-1000)$.

\section{DISCUSSION}




\subsection{Comparison with other simulations}

\subsubsection{Microlensing Simulations}

Here we discuss several other recent microlensing simulations and compare them to PopSyCLE. Kerins et al. (2009) generated synthetic maps of the microlensing optical depth, event rate, and average Einstein crossing time of the Galactic bulge, incorporating a 3-D extinction map. The GULLS code of Penny et al. (2013, 2019) improved upon this work by taking into account the effects of blending, while the MaB $\mu \mathrm{LS}$ code of Awiphan et al. (2016) also included low-mass stars and brown dwarfs. All three of these simulations follow a similar method, as summarized in P19, of drawing sources and lenses from a distribution described by the Besançon model, then assigning weights proportional that sourcelens pair's contribution to the total event rate along that sight line. A constant correction factor to adjust for the number of sources and optical depth of the Galactic bulge as derived from the Besançon model is also included to adjust the normalization of the event rate. The Besançon models used includes white dwarfs; however, neutron stars and black holes are not part of the models.

In particular, the GULLS code has been applied toward exoplanet microlensing survey designs for the Euclid and WFIRST missions. Toward this end, it models single planets orbiting a single host star. Properties of the detector and filters are included in the simulation, and GULLS can also generate images and lightcurves which includes Gaussian noise. Planetary detections were then evaluated on a $\Delta \chi^{2}$ criterion as is commonly done in other exoplanetary microlensing surveys and simulations. However, there are discrepancies between the simulation and observed event rates, as there is substantial uncertainty in the Galactic models used. As one of the purposes of GULLS is to estimate yields for microlensing survey missions, the results are rescaled by a factor to match observed star counts and optical depth (Penny et al. 2013) or star counts and event rates (P19).

In comparison, PopSyCLE is designed to understand how changes in the stellar and compact object population and imposition of observational selection criteria modify the underlying and observed microlensing event distribution. It is modular in design to allow different initial-final mass relations, dust maps, observational cuts, etc. to be used. PopSyCLE is also unique in its emphasis for studying lensing by massive compact objects, specifically black holes, and its calculation of not just photometric, but also astrometric microlensing quantities. The current version of PopSyCLE is not designed to generate lightcurves including complexities such as detector noise or atmospheric seeing, nor is it de- signed/optimized to probe the short- $t_{E}$ end of the distribution, as we are interested in the long- $t_{E}$ events. Like GULLS, PopSyCLE also has a discrepancy in the event rates as compared to observations. However, we choose to not rescale our event rates to match observation. Although this might limit PopSyCLE's predictive power, we consider the discrepancies to clearly indicate limitations in our understanding of the physics of the simulations, corrections to our data, or both (e.g. Section 5.1 of Awiphan et al. (2016)). For this reason we present our WFIRST BH yield as an order-of-magnitude estimate. However, the PopSyCLE simulation results are available for download and can be rescaled as desired for survey yield predictions.

\subsubsection{Compact Object Population Synthesis}

There are several other compact object population synthesis packages. For example, a widely used package is the Stellar EVolution $N$-body (SEVN) code $^{6}$, which combines a single stellar evolution code along with several core-collapse supernovae models, pair-instability and pair-instability pulsational supernovae, along with many different binary evolution recipes (Spera et al. 2015). We note SEVN is purely a population synthesis model, that can (and has) been interfaced with N-body stellar dynamics codes, to study interactions in star clusters, for instance. It does not include a full model of the Milky Way, nor microlensing. One thing to note is that the IFMRs in SEVN are heavily simplified analytic models that do not incorporate explosion physics. Ultimately, it would still be worth adding support for SEVN inside PopSyCLE in addition to the current PyPopStar stellar evolution code; however, we chose PyPopStar initially due to its support for a larger range of models, flexibility, and Python implementation.

\subsection{Comparison with on-sky microlensing surveys}

We advocate for defining a microlensing event using quantities that are directly observable/measured rather than fit. This is described in Dominik (2009) as a reparametrization when fitting microlensing events. For example, in the survey papers discussed, cuts were made on events whose source magnitude, $m_{S}$ is fainter than some value, where $m_{S}$ is generally set by the magnitude limit of the telescope and camera. However, the more easily observed quantity is the baseline magnitude $m_{\text {base }}$ since $m_{S}$ is generally a parameter derived from fitting and is subject to degeneracy with other parameters (Dominik 2009). When fitting light curves, blending is degenerate with the observed timescale and peak

\footnotetext{
${ }^{6}$ http://web.pd.astro.it/mapelli/group.html
} 
magnification of the event, and if blending is not taken into account correctly the microlensing parameters derived will be incorrect; masses and timescales will be systematically underestimated (Di Stefano \& Esin 1995; Woźniak \& Paczyński 1997; Han 1999). In other words, events with small $t_{E}$, large $u_{0}$, and large $b_{S F F}$ are degenerate with events with large $t_{E}$, small $u_{0}$, and small $b_{S F F}$ (Sumi et al. 2011). In order to facilitate easier comparison between simulations and observations and explore this degeneracy, we recommend that future microlensing analyses adopt cuts using observable quantities, as advocated for in Dominik (2009). However, we also suggest that such observational cuts be applied for sample selection, as these are more easily reproduced and are less dependent on differences in fitting codes and prior assumptions.

Additionally, although the mean Einstein crossing time $\left\langle t_{E}\right\rangle$ is the most commonly reported parameter by surveys, the $t_{E}$ distribution is asymmetric in linear $t_{E}$ bins $^{7}$; thus, the mean is easily skewed and depends on the range of $t_{E}$ used to estimate the mean. For future comparisons across observational surveys and simulations, the median is a better choice and is less impacted by particular cuts. It is also important to note the range of $t_{E}$ over which the distribution is made (e.g. as in Sumi et al. (2013)).

\subsection{Hunting for $\mathrm{BHs}$}

Over the years, many types of follow-up observations have been proposed and used to constrain lens properties. Agol \& Kamionkowski (2002) proposed to constrain lens masses of $\mathrm{BH}$ microlensing candidates by searching for their X-ray emission due to accretion from the interstellar medium or stellar winds. Nucita et al. (2006) and Maeda et al. (2005) used XMM-Newton and Chandra observations, respectively, to search for $\mathrm{X}$ rays from the extremely long-duration $\mathrm{BH}$ candidates MACHO-96-BLG-5 (Mao et al. 2002); however, no significant detections were made.

HST high-resolution imaging follow-up of BH candidates has also been used to measure the degree of blending (Bennett et al. 2002). As discussed in \$7.2.1, this is not particularly useful for selecting photometric candidates for astrometric follow-up; however, it still may be a useful means of confirming that the lens is not a massive star. Poindexter et al. (2005) also suggested that HST observations could be used to measure the source proper motion; however they note that many degeneracies remain even with this measurement. High-resolution im-

\footnotetext{
${ }^{7} t_{E}$ is often plotted in bins of $\log t_{E}$ which makes the distribution look more symmetric.
}

ages can be taken many years after an event, when it may be possible to resolve the source and lens (Batista et al. 2015; Bennett et al. 2015; Alcock et al. 2001, Abdurrahman et al. in prep.) and the absence of a lens may provide additional confirmation of a $\mathrm{BH}$.

For nearby sources, it is sometimes also possible to measure the source distance via parallax, which would give complete event parameters. The combination of photometry and astrometry is powerful not only for $\mathrm{BH}$ lenses, but also for obtaining precise mass measurements of any type of lens, as was shown with the first astrometric microlensing signal detected outside our Solar System (Sahu et al. 2017). Another method of breaking degeneracies is to have the ability to resolve the images themselves, which is possible for stellar-mass lenses using interferometric techniques (Dong et al. 2019).

We have not yet considered the number of BHs detectable with the Gaia satellite, an astrometric space mission which has been operating since 2013 (Gaia Collaboration et al. 2016). Although Gaia has incredible astrometric precision for parallax measurement (down to $\sim 10 \mu$ as for some stars by the end of the mission), it does not perform as well for single-epoch astrometric measurements in the bulge due to the decreased observing cadence as well as significant stellar crowding $(>1$ mas, Rybicki et al. (2018)). Additionally, Gaia observes at green optical wavelengths, which cannot probe dusty, high extinction regions like the bulge. However, there are estimates of the number of BHs that Gaia will be able to detect over it's lifetime. Mashian \& Loeb (2017) estimated that $\sim 2 \times 10^{5} \mathrm{BHs}$ in astrometric binary systems can be detected over Gaia's 5 year mission; however, this is a severe overestimate, as this calculation has neglected extinction and crowding effects. Rybicki et al. (2018) estimated that a few isolated stellar mass BHs should be detectable astrometrically at the end of the Gaia mission. Wyrzykowski \& Mandel (2019) used Gaia DR2 data to calculate distances and proper motions for sources in OGLE-III microlensing events from 2001 to 2009, providing additional information to perform a more careful reanalysis of the lens masses to determine whether they could be BHs.

In $\S 7$, two different methods to better understand BHs in the Milky Way are discussed. The first involves using a combination of astrometry and photometry to measure $t_{E}, \pi_{E}$, and $\delta_{c, \max }$, which allows lens masses to be measured for individual microlensing events. The second involves photometry only to measure $t_{E}$ and $\pi_{E}$, enabling statistical constraints on a population of $\mathrm{BHs}$. Although the $\mathrm{BH}$ nature of individual lenses cannot be confirmed, ensemble information can still be gleaned. To obtain masses with the second method would in- 
volve assuming some type of Galactic model or spatial distribution. It is interesting to note that searching for a small/undetectable parallax signal to identify $\mathrm{BHs}$ is the opposite of previous approaches in the literature (Poindexter et al. 2005; Wyrzykowski et al. 2016; Drlica-Wagner et al. 2019).

\subsection{Future Work}

In the next version of PopSyCLE, we plan to add support for stellar and compact object binary systems, compact object mergers, metallicity-dependent IFMRs, different compact object spatial distributions, and primordial BHs. This will allow for a more realistic simulation and a larger exploration of parameter space. The lack of these features at the present put some caveats on this work, which we discuss.

\subsubsection{Binarity and Mergers}

Although all of the comprehensive catalogs of microlensing events in the literature from which event rates and optical depths are calculated are only for events which can be fit by point source point lens (PSPL) models (Sumi \& Penny 2016; Wyrzykowski et al. 2015; Mróz et al. 2017), it is estimated that that binary lenses will consist of around 10\% of Galactic bulge stars lensing events (Mao \& Paczynski 1991). It is difficult to ascertain the binary fraction from the observed distribution of microlensing events because binary lenses can produce lightcurves that resemble single lens events. For a widely separated binary, only one of the lenses might be lensing the source star, while for a closely separated binary, both lenses act as a single more massive lens. Moreover, the addition of another lens greatly increases the complexity and variety of lightcurve shapes, depending on how the source approaches and/or crosses the caustics, making binary lens events difficult to identify. Additionally there is the issue of binary source stars. Similar to binary lens events, binary source events can produce lightcurves that appear quite similar to PSPL events (Dominik 1998; Han \& Jeong 1998), although these degeneracies can be broken by the addition of astrometric information (Nucita et al. 2016).

Galaxia does not have support for binaries; however, most massive stars are in binary systems (Duchêne \& Kraus 2013). Additionally, our current population synthesis method does not include compact object binaries (either compact-compact or compact-stellar). PyPopStar currently has a heuristic prescription for stellar multiplicity, where stars are taken from single-stellar evolution models and combined into multiple systems based on multiplicity fraction. However, this does not take into account the effect that mass exchange has on the evolution of stars in multiple systems. For main sequence stars, such effects are likely negligible; however, in the later stages of stellar evolution, binary evolution cannot be neglected. For a more rigorous treatment of stellar multiplicity, binary evolution models will be incorporated into PyPopstar. Additionally, future work on binary IFMRs will also be added to PyPopStar.

Adding support for binaries (stellar-stellar, compactstellar, and compact-compact) into PopSyCLE would help us put further constraints on the number of BHs in the Milky Way and understand BH formation channels. For example, both single and binary BHs may form from binary star systems; single star systems can be formed when the stellar binary is disrupted or merges. If the binary is disrupted, this could impart large kick velocities to the BHs; the frequency at which disruption occurs depends on the assumptions made about the kicks (Wiktorowicz et al. (2019) and references therein). The spatial distribution will also be affected by disrupted binaries and the associated kicks; the scale height distribution of X-ray binaries can act as a proxy for different compact object formation mechanisms (Repetto et al. 2017).

Another possibility for forming more massive stellar mass BHs is through the mergers of less massive BHs. By adjusting the merger rate and fraction, we can adjust the mass spectrum and fraction of BHs in single and binary systems. Adding support for compact-object mergers would allow us determine our ability to set constraints on the merger rate and fraction, which would again improve our understanding of $\mathrm{BH}$ formation channels and LIGO merger events.

\subsubsection{Metallicity}

When performing population synthesis, metallicity has not been taken into account. Although Galaxia and PyPopStar have metallicity support, the R18 IFMR does not. With the IFMR, metallicity can have a significant effect on the mass and type of the compact remnant. In particular, having $[\mathrm{Fe} / \mathrm{H}]>[\mathrm{Fe} / \mathrm{H}] \odot$ will not particularly change the IFMR, while having $[\mathrm{Fe} / \mathrm{H}]<$ $[\mathrm{Fe} / \mathrm{H}]_{\odot}$ will produce more high-mass black holes. New models to be used in the IFMR have been run to include a metallicity dependence (T. Sukhbold, private communication.) A non-mutually exclusive option would be to add other IFMRs that have a dependence on both the progenitor mass and metallicity, such as the one in Appendix C of Spera et al. (2015).

In Galaxia, the metallicity of the bulge population is centered at solar metallicity with a spread $\sigma_{[\mathrm{Fe} / \mathrm{H}]}=0.4$ (Table 2, Sharma et al. (2011)), and with respect to the current iteration of PopSyCLE, about $80 \%$ of stars within 
$10 \mathrm{kpc}$ of Earth in the bulge direction have $-0.5<$ $[\mathrm{Fe} / \mathrm{H}]<0.5$, where the IFMR likely does not change significantly. Thus, the solar-metallicity approximation is reasonable for the bulk statistics; the largest effect would be a slight deficit of higher-mass BHs.

\subsubsection{Compact object distributions and kinematics}

In PopSyCLE, it is assumed that the positions of the compact objects followed the stellar spatial distribution. Additionally, the velocity of the object is assumed to be the stellar progenitor velocity and kick velocity added together; however, this is only true at the time the compact object is born. This choice is roughly justified given that the number of objects leaving a region is the same as the number of objects entering that region, since the direction of the kick velocity is random. However, effects like dynamical friction might cause black holes to settle toward the Galactic Center. Conversely, supernovae may provide large enough kicks to unbind neutron stars from the Galactic disk. In future iterations of PopSyCLE, we will allow for different compact-object spatial distributions. Currently, the kick velocities for both NSs and BHs is tunable; however, it is only allowed to be a single value. In the future, support for kick velocity distributions will be added.

The additions above are necessary to provide support for primordial black holes (PBHs) in PopSyCLE. Ultimately, we plan to implement a PBH mass spectrum such that PBHs can be injected with their own position, velocity, and mass distributions that differ from the underlying stellar halo (Carr et al. 2016; Chapline \& Barbieri 2018). Similar to the IFMR, a variety of different mass spectra could be implemented.

\section{CONCLUSIONS}

We have developed a Milky Way microlensing simulation, dubbed PopSyCLE, which is the first to consider both photometric and astrometric microlensing effects and perform compact object population synthesis in a realistic manner. With PopSyCLE, we investigate different strategies to hunt for isolated stellar mass BHs in the Milky Way and measure their masses. We highlight the following results:

- Assuming a state-of-the-art initial-final mass relations, the BH present-day mass function has structure (peaks and gaps) that can be measured with samples of $\mathcal{O}(100) \mathrm{BH}$ mass measurements from both LIGO detections and $\mathrm{BH}$ microlensing surveys.

- The optimal isolated BH candidates to follow up astrometrically are long-duration microlensing events with Einstein crossing times $t_{E} \gtrsim 90-120$ days; additional selection criteria based on the source flux fraction $b_{S F F}$ or the impact parameter $u_{0}$ or obtaining high-resolution imaging does not significantly improve the outcome. Current photometric surveys and astrometric follow-up campaigns should yield a $40 \%$ success rate for measuring $\mathrm{BH}$ masses.

- From photometry alone, BHs can be identified in a statistical manner with a combined measurement of $t_{E}$ and the microlensing parallax $\pi_{E}$. The BH detection rate can be raised to $\sim 85 \%$ by selecting events with both $t_{E}>120$ days and $\pi_{E}<0.08$.

- $\mathrm{BH}$ lenses are easily distinguished from stellar lenses with a combined measurement of the microlensing parallax $\pi_{E}$ and the maximum astrometric shift $\delta_{c, \max }$, providing a useful method for confirming the $\mathrm{BH}$ nature of the lens and measuring its mass. In particular, we note that $\mathrm{BH}$ lenses nearly always have $\pi_{E}<0.1$.

- The WFIRST microlensing survey will be able to measure the masses of $\mathcal{O}(100-1000)$ isolated $\mathrm{BHs}$ over it's 5 year lifetime, which is at least an order of magnitude more than is possible with individual astrometric follow-up. This is sufficient to constrain the BH IFMR, binary fraction, and kick velocity distribution.

PopSyCLE can also be used to forward model microlensing survey results and constrain the properties of compact objects, the initial-final mass relation, Galactic structure, the existence of primordial black holes, and more. PopSyCLE can be downloaded from https:// github.com/jluastro/PopSyCLE and community contributions are welcome. We also provide the simulation files used for this work at https://drive.google.com/ open?id=12ALPCqMVOBN54fy1YhHfiJjI9Y1bnlQj.

\section{ACKNOWLEDGEMENTS}

We thank the PALS collaboration and Will Clarkson for helpful conversations. We also thank the anonymous referee for comments that improved this paper. C. Y. L. and J. R. L. acknowledge support by the National Aeronautics and Space Administration (NASA) under Contract No. NNG16PJ26C issued through the WFIRST Science Investigation Teams Program. Portions of this work were performed under the auspices of the U.S. Department of Energy by Lawrence Livermore National Laboratory under Contract DE-AC5207NA27344 and supported by the LLNL-LDRD Program under Project No. 17-ERD-120. 
Software: Galaxia (Sharma et al. 2011), astropy (Astropy Collaboration et al. 2013, 2018), MIST v1.2 (Choi et al. 2016; Dotter 2016), Matplotlib (Hunter 2007, DOI:10.1109/MCSE.2007.55), NumPy (van der
Walt et al. 2011, DOI:10.1109/MCSE.2011.37 ), SciPy (Virtanen et al. 2019), PyPopStar (Hosek et al. in prep, https://github.com/astropy/PyPopStar), PopSyCLE (this work, 10.5281/zenodo.3464129).

\section{APPENDIX}

\section{A. GALAXIA GALACTIC MODEL}

There are several known issues in the Besançon model, and by extension Galaxia, particularly with the bulge. Many of these are discussed in $\S 6.2$ of P19, albeit for a slightly different version of the Besançon model than that implemented in Galaxia. We modify the bulge kinematics of Galaxia, specifically the pattern speed and velocity dispersions, in an attempt to ameliorate these issues. We also validate these changes by performing some comparisons to star counts and event rates from Mróz et al. (2019) and Sumi \& Penny (2016).

\section{A.1. Galactic Models Comparison}

There are three specific properties of the bulge we consider modifying:

- Pattern speed: Galaxia implements a pattern speed of $\Omega=71.62 \mathrm{~km} / \mathrm{s} / \mathrm{kpc}$ (see $\S 3.3$ in Sharma et al. (2011)). However, this is nearly twice as fast as the most recent values reported in the literature, which range from around 36 to $44 \mathrm{~km} / \mathrm{s} / \mathrm{kpc}$ (Bovy et al. 2019; Clarke et al. 2019; Sanders et al. 2019), determined using combinations of Gaia DR2, VVV, and APOGEE.

- Velocity dispersion: Galaxia implements velocity dispersions of $\sigma_{R}=\sigma_{\phi}=110 \mathrm{~km} / \mathrm{s}$. However, this produces microlensing events with timescales that are too short, which suggests smaller velocity dispersions might be more appropriate. $^{8}$ In reality, $\sigma_{R}$ and $\sigma_{\phi}$ should vary with latitude and longitude (Howard et al. 2008, 2009), but to implement this in Galaxia would require significant rewriting of the code, which is far beyond the scope of this current work.

- Bar angle and length: Galaxia implements a bar angle of $\alpha=11.1^{\circ}$, where $\alpha$ is the angle from the Sun-Galactic Center line-of-sight, and a major axis scale length of $x_{0}=1.59 \mathrm{kpc}$. It is suggested that the angle should be closer to $\alpha=28^{\circ}$ (Wegg \& Gerhard 2013; Wegg et al. 2015), with a shorter scale length of $0.7 \mathrm{kpc}$. In fact, Portail et al. (2017) performed dynamical modeling using $\alpha=28^{\circ}$ to find a bar pattern speed of around 40 $\mathrm{km} / \mathrm{s} / \mathrm{kpc}$. However, there is still considerable debate in the literature about the value of $\alpha$ and the scale length, with values for $\alpha$ ranging from $10^{\circ}-45^{\circ}$ (see Robin et al. (2012) for a summary and references).

We create two new variations of the original Galactic model implemented in Galaxia. We dub "v2" to be the version of Galaxia with a pattern speed $\Omega=40 \mathrm{~km} / \mathrm{s} / \mathrm{kpc}$ and bulge velocity dispersions $\sigma_{R}=\sigma_{\phi}=100 \mathrm{~km} / \mathrm{s}$. We dub "v3" to be the version where the bar is short and tilted, with $\Omega=40 \mathrm{~km} / \mathrm{s} / \mathrm{kpc}$, bulge velocity dispersions $\sigma_{R}=\sigma_{\phi}=100$ $\mathrm{km} / \mathrm{s}$, bar angle $\alpha=28^{\circ}$ and major axis scale length $x_{0}=0.7 \mathrm{kpc}$.

Tables 5 and 7 compare PopSyCLE stellar densities, event rates, and Einstein crossing times to results from SP16 and M19 for v2 and v3, respectively. Figure 7 summarizes the results of the two tables together. The stellar densities of v2 match reasonably well with the observed number counts; v3 is consistently too low. Note that although in projection the length of the bar is the same, as $\sin \left(11.1^{\circ}\right) \cdot 1.59 \mathrm{kpc} \approx \sin \left(28^{\circ}\right) \cdot 0.7 \mathrm{kpc}(\mathrm{P} 19)$, the number counts are quite different, due to extinction. The event rates for v3 match reasonably well with the observed rates; v2 is consistently too high. With respect to the average Einstein crossing time, it is not as clear whether v2 or v3 matches the observed values better.

Based on this analysis we chose to use v2 instead of v3 for the analysis in the main text of this paper. Stellar density is the more fundamental observational quantity, hence we prefer the simulation reproduce this aspect accurately. The event rate is microlensing-specific and dependent on many more factors (e.g., the detection efficiency correction).

\footnotetext{
8 This issue of too short microlensing events is also a problem described in $\S 6.2 .2$ of Penny et al. (2019), who implement the Besançon model to study microlensing, although it should be noted that the versions of the Besançon model in Penny et al. (2019) and Galaxia are slightly different.
} 


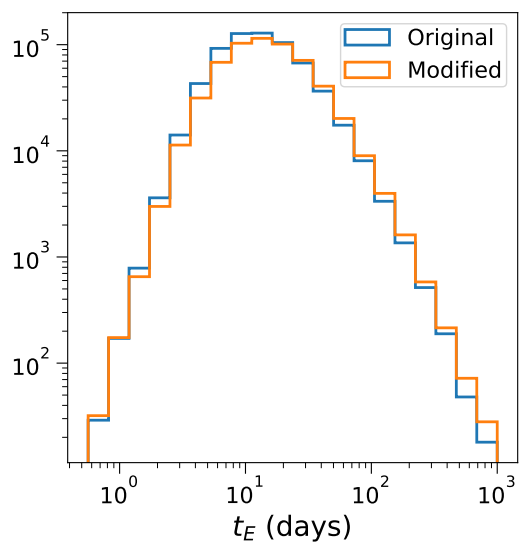

Figure 16. $t_{E}$ distribution of microlensing event candidates (i.e. without any selection cuts applied). The "Original" curve was generated using Galaxia unmodified. The "Modified" curve was generated using Galaxia v2 with $\Omega=71.62 \mathrm{~km} / \mathrm{s} / \mathrm{kpc}$ and $\sigma_{R}=\sigma_{\phi}=110 \mathrm{~km} / \mathrm{s}$. It can be seen that the modified Galaxia has more long $t_{E}$ events and fewer short $t_{E}$ events.

Table 7. Comparing PopSyCLE to surveys (Galaxia v3)

\begin{tabular}{|c|c|c|c|c|c|c|}
\hline \multirow[t]{2}{*}{ Field } & \multicolumn{2}{|c|}{$\mathrm{n}_{s}\left(10^{6} / \mathrm{deg}^{2}\right)$} & \multicolumn{2}{|c|}{$\Gamma\left(10^{-6} /\right.$ star $\left./ \mathrm{yr}\right)$} & \multicolumn{2}{|c|}{$\left\langle t_{E}\right\rangle$ (days) } \\
\hline & Obs. & Mock & Obs. & Mock & Obs. & Mock \\
\hline $\mathrm{F} 00^{M 19}$ & 2.62 & 1.48 & $1.3 \pm 0.8$ & 4.35 & 32.6 & 14.8 \\
\hline $\mathrm{F} 01^{M 19}$ & 4.54 & 2.04 & 5.5 & 3.69 & 39.5 & 46.2 \\
\hline $\mathrm{F}_{0} 3^{S}$ & 3.64 & 2.47 & $14.0_{-2.4}^{+2.9}$ & 8.26 & 25.5 & 20.3 \\
\hline $\mathrm{F} 11^{M 19}$ & 4.95 & 3.66 & $16.2 \pm 1.3$ & 17.88 & 21.8 & 19.7 \\
\hline $\mathrm{F} 12^{M 19}$ & 3.2 & & & 2.8 & 36.7 & 51.1 \\
\hline $\mathrm{F} 13^{M 19}$ & 4.51 & 2.19 & $5.2 \pm 1.1$ & 4.9 & 30.8 & 28.2 \\
\hline
\end{tabular}

Note-Identical analysis as presented in Table 5 , but using Galaxia v3 (with the tilted shorter bar) instead of v2. Fields with ${ }^{M 19}$ indicate the observed values come from M19, while those with ${ }^{S}$ are from SP16.

However, it is curious in and of itself that the tilted bar creates agreement in one regime but not the other. Additional modifications (such as the 3-D $E(B-V)$ map) may be necessary to bring the models into better agreement with observation. We do not explore this further here, and leave detailed Galactic modeling to the investigation of future work. However, it is worth noting how this uncertainty affects some of the results of this paper. In $\$ 7.2 .1$, the fraction of $\mathrm{BH}$ events at long times can be up to a factor of 2 higher for v3 than for v2. In $\S 7.5$ we consider the number of BH masses WFIRST can measure; the number is about a factor of 4 higher for v2 than for v3.

The rest of the analysis and validation performed in this appendix is also using v2.

\section{A.2. Bulge Kinematics}

Following $\S 6.2 .2$ of P19, we compare the results of our simulation to observational studies of bulge kinematics. In Clarkson et al. (2008), a study of proper motions in the Galactic Bulge is performed using observations of the HST SWEEPS field (centered at $(l, b)=(1.25,-2.65)$ covering an area of $11 \operatorname{arcmin}^{2}$ ) in the HST F606W and F814W bands. To compare, we created a synthetic survey in the same direction of the same area using Galaxia; we use I and $\mathrm{R}$ band as these have the closest effective wavelength as the HST F606W and F814W bands. First, we select stars in Galaxia photometrically (Figure A.2) similarly to Clarkson et al. (2008) to obtain a red population (bulge proxy) and a blue population (disk proxy). We obtain 347 blue stars and 699 red stars (c.f. P19 37 blue stars and 105 red stars, drawing from an area of $\left.1.44 \operatorname{arcmin}^{2}\right)$. 

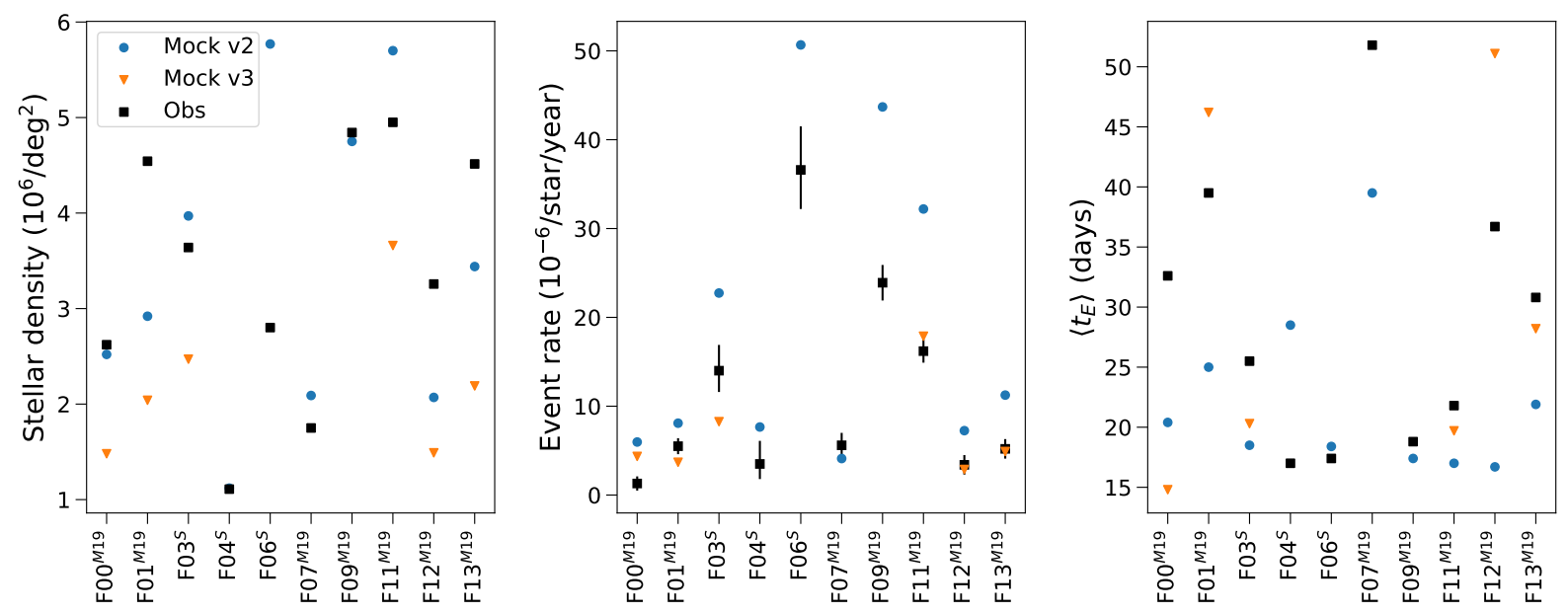

Figure 17. Comparison of the stellar density, event rate, and average Einstein crossing times for several fields. The labels FXX correspond to the field used (see Table 3), and the superscript correspond to the paper from which observed values and selection criteria for the mock values were drawn (S from Sumi \& Penny (2016), M19 from Mróz et al. (2019)). Note that only a handful of fields were initially tested, which is why there are certain fields for v3 that have no points.

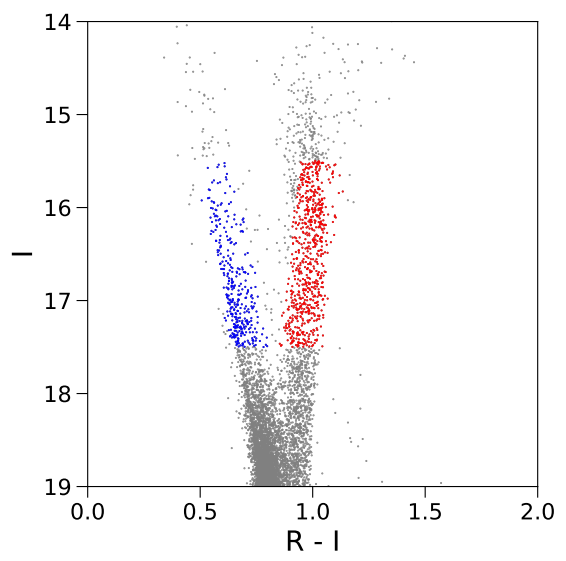

Figure 18. Photometrically selected red and blue populations, c.f. Clarkson et al. (2008) Figures 8 and 9 . The gray points correspond to all stars, while the red points correspond to the proxy bulge population and the blue points correspond to the proxy disk population.

Table 8. Clarkson et al. (2018), BGM1106, Galaxia Proper Motion Comparison

\begin{tabular}{lcccccc}
\hline \hline \multicolumn{1}{c}{ Model/Data } & $\Delta \mu_{l *}$ & $\Delta \mu_{b}$ & $\sigma_{l *}$, blue & $\sigma_{b}$, blue & $\sigma_{l *}$, red & $\sigma_{b}$, red \\
& \multicolumn{7}{c}{} & & & & \\
\hline Clarkson et al. (2008) & $3.24 \pm 0.15$ & $-0.81 \pm 0.12$ & 2.2 & 1.3 & 3.0 & 2.8 \\
BGM1106 (P19) & $3.53 \pm 0.65$ & $-0.12 \pm 0.32$ & $2.47 \pm 0.29$ & $1.11 \pm 0.13$ & $5.19 \pm 0.36$ & $2.64 \pm 0.18$ \\
Galaxia & 4.51 & -0.42 & 2.48 & 1.42 & 3.74 & 2.32 \\
\hline
\end{tabular}

Note-All units are (mas/yr). $\Delta$ is defined as blue - red.

We then compare our results to those of Clarkson et al. (2008) and P19, who use a different version of the Besançon model, named BGM1106 for short. The results are summarized in Table 8, and illustrated in Figures A.2 and A.2. In particular, the match between Clarkson et al. (2008) is improved in $\sigma_{l, *}$ for the red population. 

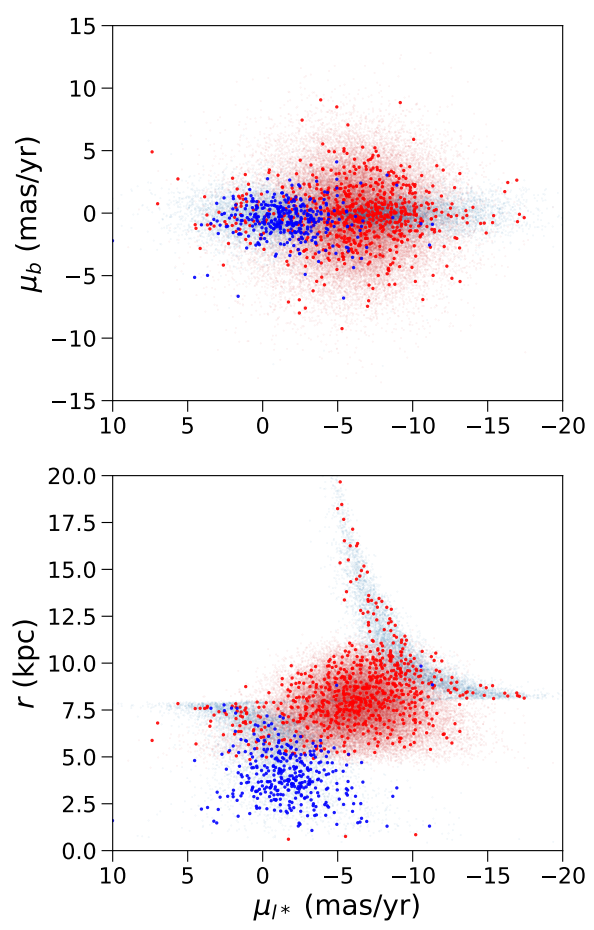

Figure 19. Large red points are the photometrically selected bulge stars ("red"/bulge proxy) while large blue points are the photometrically selected disk stars ("blue"/disk proxy). Small red points are the bulge stars and small blue points are the disk stars in Galaxia. Top: Proper motion vector point diagram for the red and blue populations, c.f. P19 Figure 20. Bottom: Distance vs. longitudinal proper motion diagram for the red and blue populations, c.f. P19 Figure 20.
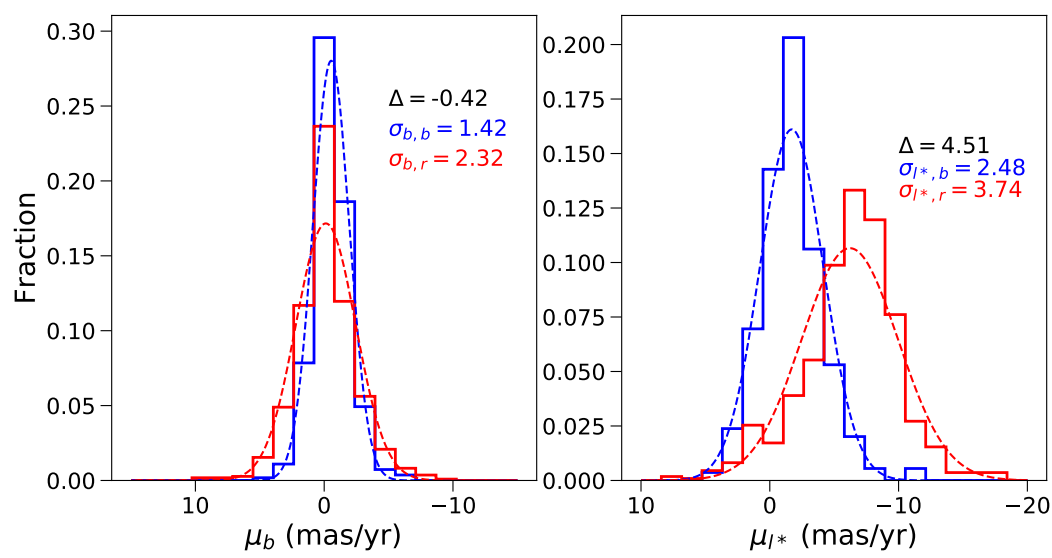

Figure 20. Histograms of proper motion for the red (bulge-proxy) and blue (disk-proxy) populations from Galaxia, with the dotted line being a Gaussian of the mean and standard deviation of each respective population. The $\Delta$ listed gives the differences between the means of the populations (blue - red). Units of all inset numbers are mas/yr.

\section{B. EXTINCTION}

The amount of extinction given in Galaxia, which uses the Schlegel et al. (1998) dust map overestimates the extinction towards the Galactic Bulge. Thus, in PopSyCLE we have instead chosen to implement the reddening law of Damineli et al. (2016), which is tailored for the direction toward the Galactic Plane. We continue to use the color 
excess values $E(B-V)$ from Schlegel et al. (1998), which is not strictly correct; however, this is satisfactory, as we show below.

We compare the Galaxia model using the $E(B-V)$ from Schlegel et al. (1998) with either the Schlegel et al. (1998) or Damineli et al. (2016) reddening laws, to the data from the OGLE Early Warning System (EWS). From the OGLE 2017 EWS, 4 different events were selected: OGLE-2017-BLG-0001 at (l,b) = (0.92, -1.63); OGLE-2017-BLG-0100 at (-2.07, 0.98); OGLE-2017-BLG-0150 at (1.74, -4.46); OGLE-2017-BLG-0921 at (-0.71, -1.79). These 4 particular events were selected because their finding charts had different CMDs. For these events, the $I$ and $V$ magnitudes of stars in a $2^{\prime} \times 2^{\prime}$ area centered on the event are provided. With Galaxia, fields in those directions are generated with that equivalent area $\left(0.0011 \mathrm{deg}^{2}\right)$. Since Galaxia returns the distance and absolute magnitude of the stars in various filters, along with and the Schlegel E(B-V) color excess, the apparent magnitude of these stars using either the Schlegel or Damineli reddening can be calculated and used to produce synthetic CMDs and luminosity and color functions. The comparisons are plotted in Figure B.

In general, Galaxia captures the various structures in the OGLE CMDs. Although there is still some underestimation in the number of stars, the Damineli et al. (2016) reddening law does a significantly better job than Schlegel et al. (1998) at capturing the total number of stars in the field. The Damineli et al. (2016) law also does slightly better at replicating the CMD shape and luminosity and color functions.
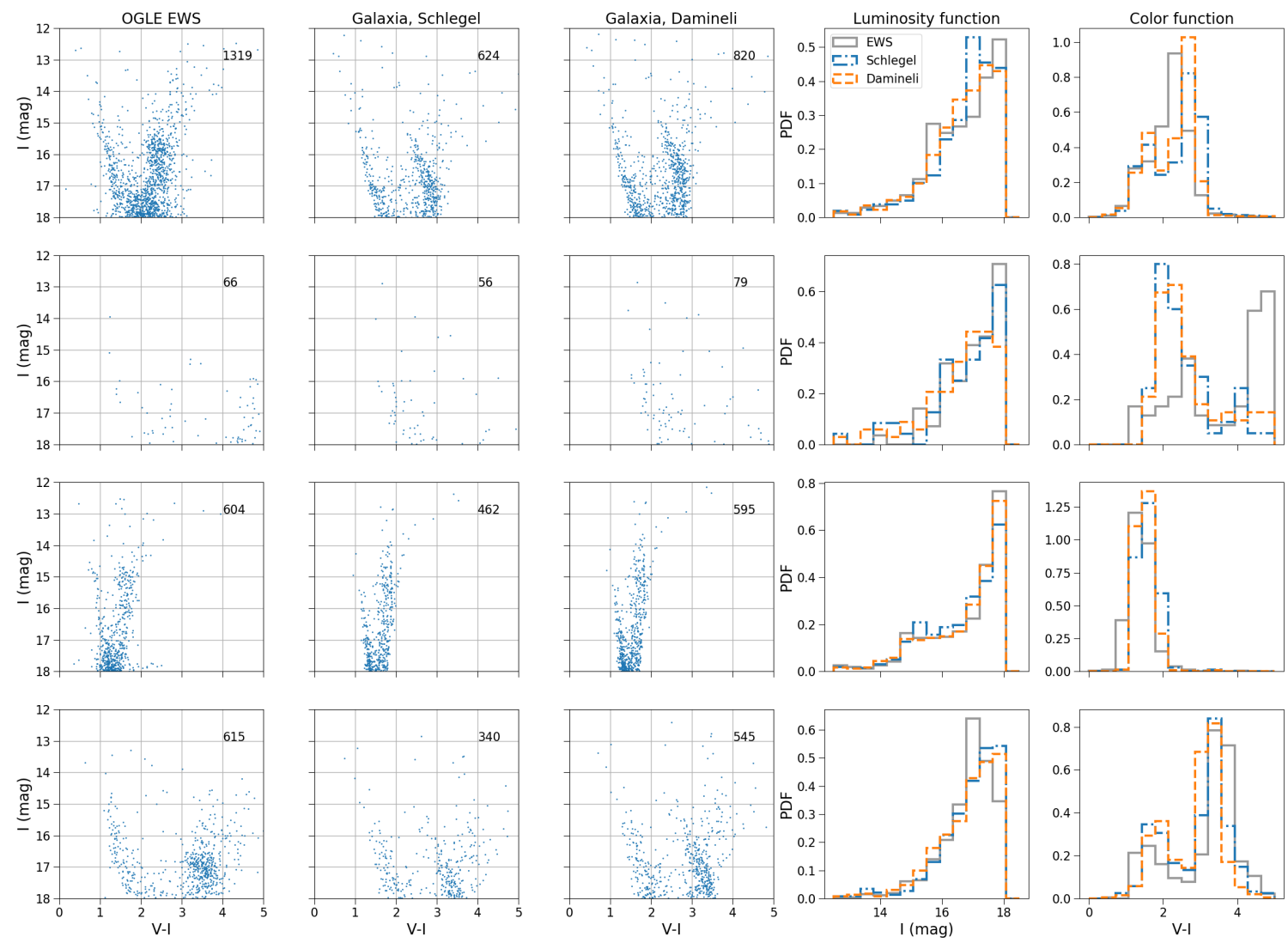

Figure 21. Comparison of CMDs, luminosity, and color functions for different fields and extinction laws. The rows, from top to bottom, correspond to the fields OGLE-2017-BLG-0001 at $(1, \mathrm{~b})=(0.92,-1.63)$, OGLE-2017-BLG-0100 at $(1, \mathrm{~b})=(-2.07$, 0.98), OGLE-2017-BLG-0150 at $(1, b)=(1.74,-4.46)$, and OGLE-2017-BLG-0921 at $(1, b)=(-0.71,-1.79)$. The numbers in the corners of the CMDs correpond to the number of stars in that CMD.

We also consider an extinction map produced by Nataf et al. (2013) using data from the OGLE-III, VVV, and 2MASS surveys. However, for the fields available, the differences are random and small, hence they do not consistently skew our number counts in one direction. 


\section{INITIAL-FINAL MASS RELATION}

As defined in R18 Equations (1) through (4), the black hole initial-final mass relation (IFMR) is a piecewise function, where the two pieces are dubbed Branches I and II. Branch I, which covers $15 \leq M_{Z A M S} \leq 40 M_{\odot}$, the remnant $\mathrm{BH}$ mass is given by

$$
M_{B H}\left(M_{Z A M S} ; f_{e j}\right)=f_{e j} M_{B H, c o r e}\left(M_{Z A M S}\right)+\left(1-f_{e j}\right) M_{B H, \text { all }}\left(M_{Z A M S}\right)
$$

where $f_{e j}$ is the ejection fraction describing how much of the envelope is ejected in a supernova explosion $\left(f_{e j}=0\right.$ is where the entire star collapses, $f_{e j}=1$ is where only the star's He-core collapses), and $M_{B H \text {,core }}$ and $M_{B H, \text { all }}$ are defined as

$$
\begin{aligned}
M_{B H, \text { core }}\left(M_{Z A M S}\right) & =-2.049+0.4140 M_{Z A M S} \\
M_{B H, \text { all }}\left(M_{Z A M S}\right) & =15.52-0.3294\left(M_{Z A M S}-25.97\right)-0.02121\left(M_{Z A M S}-25.97\right)^{2}+0.003120\left(M_{Z A M S}-25.97\right)^{3} .
\end{aligned}
$$

In PopSyCLE we use $f_{e j}=0.9$ as this is the value reported by R18 that most closely reproduces the observed distribution of BH masses. The $M_{B H \text {,core }}$ term describes the BH IFMR where only the star's He-core collapses, while the $M_{B H \text {,all }}$ term describes the BH IFMR where the entire star collapses; $f_{e j}$ interpolates between the two. The black hole IFMR for Branch II, which covers $45 \leq M_{Z A M S} \leq 120 M_{\odot}$, is

$$
M_{B H, \text { core }}\left(M_{Z A M S}\right)=5.697+7.8598 \times 10^{8}\left(M_{Z A M S}\right)^{-4.858} .
$$

Similarly, there is a piecewise defined neutron star initial-final mass relation. Overall, there are seven branches, Branches I through VII, described in R18 Equations (11) through (16). Each branch of the IFMR is defined over a particular range of ZAMS masses. Five of these branches are described by third order polynomials, and two are described by Gaussian distributions.

We have made the following modifications to the original initial-final mass function/relation:

1. Since the gap between $40 \leq M \leq 45 M_{\odot}$ between Branches I and II is due to the discrete sampling of the simulations from Sukhbold et al. (2016), we extend Branches I and II such that there isn't a gap. Specifically, we find where the function describing Branch I intersects Branch II, assuming $f_{e j}=0.9$; this point is $M_{Z A M S}=$ $42.21 M_{\odot}$. So for our purposes, Branch I goes from $15 \leq M_{Z A M S} \leq 42.21 M_{\odot}$ and Branch II goes from $42.21 \leq$ $M_{Z A M S} \leq 120 M_{\odot}$.

2. A distinction is made between black holes made by fallback, and those made by direct collapse. As described by Equation (8), black holes only form from direct collapse immediately after the SNe. However, for our purposes, a black hole formed by fallback vs. direct collapse is not relevant. We modify the fraction of black holes to then be

$$
X_{B H}=\frac{N_{B H}+N_{f b}}{N_{B H}+N_{N S}+N_{f b}}=1-X_{N S}
$$

We do this for completeness; however $N_{f b}$ is quite small compared to $N_{B H}$ and this will not substantially change the results.

3. Since all the neutron stars fall in such a small mass range (between 1.3 and $1.9 M_{\odot}$ ), for simplicity, we just assume the neutron star initial-final mass function is a constant, that is,

$$
M_{N S}\left(M_{Z A M S}\right)=1.6 M_{\odot}
$$

where $1.6 M_{\odot}$ was selected since is the mean of this mass range. In future versions, we plan to release a more realistic NS IFMR.

\section{INITIAL-FINAL GROUP MASS RATIO}

Galaxia simulation output is divided into groups of stars with a similar age in order to determine the appropriate number, types, and masses of compact objects for that group. Each group is treated as a simple stellar population of a fixed age and solar metallicity. Currently, differences in metallicity within each group are ignored since the R18 IFMR 
only has solar metallicity. The total stellar mass of the age group from Galaxia is the present-day group mass; however, the initial group mass is needed in order to determine how many black holes and other compact objects should be added to Galaxia. As each group ages, the present-day mass decreases in a non-linear fashion and the conversion from present-day to the initial group mass must be derived through simulations. Ultimately, during PopSyCLE simulations, this relation between the initial-final group mass ratio and age is used to estimate the total initial group mass, generate a PyPopStar cluster of that mass and age, and insert the resulting white dwarfs, neutron stars, and compact objects back into PopSyCLE.

The relationship between the initial-final group mass ratio and age is calibrated using PyPopStar by simulating groups of stars of mass $10^{7} M_{\odot}$ over a wide range of ages (Figure D). The group mass is chosen to be large, to ensure that stochasticity does not dominate the result of the initial-final group mass ratio. For all age groups, we adopt the same Kroupa IMF and MIST evolutionary models described in Section 3. The process is as follows:

A $10^{7} M_{\odot}$ group of stars is generated using PyPopStar and evolved to the desired age. As the MIST isochrones include some white dwarfs, while Galaxia does not include any white dwarfs, all stars beyond the main sequence (post-AGB and Wolf-Rayet stars) are discarded from the group. The mass of the remaining stars constitute that age group's current stellar mass. The current age group's stellar mass is then divided by the initial group's stellar mass $\left(10^{7} M_{\odot}\right)$ to obtain the initial-final group mass ratio.

Implicit in this process, it is assumed that the IMF shape and mass limits used to calculate the initial-current group mass ratio with PyPopStar are the same as the IMF used in Galaxia.

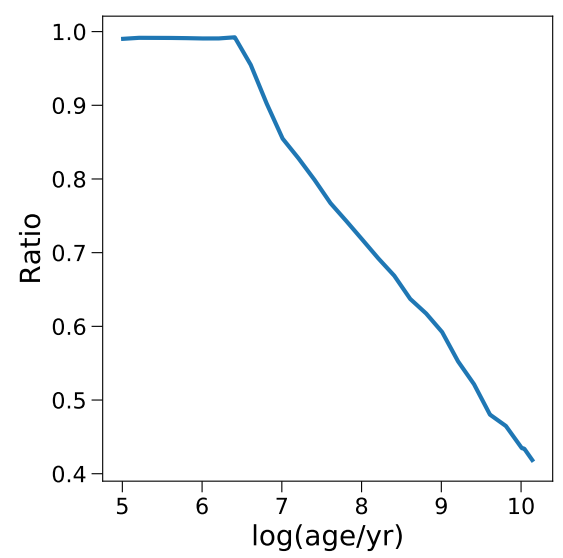

Figure 22. Ratio of current group mass to initial group mass as a function of group age. Most stars do not begin to evolve off the main sequence until after $10^{6}$ years. After that, stellar mass is converted to remnant mass in a roughly linear fashion as a function of log age.

\section{REFERENCES}

Abbott, B. P., Abbott, R., Abbott, T. D., et al. 2016, Physical Review X, 6, 041015, doi: 10.1103/PhysRevX.6.041015

Abbott, B. P., Abbott, R., Abbott, T. D., et al. 2019, Phys.

Rev. X, 9, 031040, doi: 10.1103/PhysRevX.9.031040

Agol, E., \& Kamionkowski, M. 2002, MNRAS, 334, 553, doi: 10.1046/j.1365-8711.2002.05523.x

Alcock, C., Akerlof, C. W., Allsman, R. A., et al. 1993, Nature, 365, 621, doi: 10.1038/365621a0

Alcock, C., Allsman, R. A., Alves, D. R., et al. 2000, ApJ, 542, 281, doi: 10.1086/309512
—. 2001, Nature, 414, 617, doi: 10.1038/414617a

Astropy Collaboration, Robitaille, T. P., Tollerud, E. J., et al. 2013, A\&A, 558, A33, doi: 10.1051/0004-6361/201322068

Astropy Collaboration, Price-Whelan, A. M., Sipőcz, B. M., et al. 2018, AJ, 156, 123, doi: 10.3847/1538-3881/aabc4f

Aubourg, E., Bareyre, P., Bréhin, S., et al. 1993, Nature, 365, 623, doi: 10.1038/365623a0

Awiphan, S., Kerins, E., \& Robin, A. C. 2016, MNRAS, 456, 1666, doi: 10.1093/mnras/stv2625 
Baraffe, I., Homeier, D., Allard, F., \& Chabrier, G. 2015, A\&A, 577, A42, doi: 10.1051/0004-6361/201425481

Batista, V., Beaulieu, J. P., Bennett, D. P., et al. 2015, ApJ, 808, 170, doi: 10.1088/0004-637X/808/2/170

Belczynski, K., Kalogera, V., Rasio, F. A., et al. 2008, The Astrophysical Journal Supplement Series, 174, 223, doi: 10.1086/521026

Belczynski, K., Wiktorowicz, G., Fryer, C. L., Holz, D. E., \& Kalogera, V. 2012, ApJ, 757, 91, doi: 10.1088/0004-637X/757/1/91

Bennett, D. P., Becker, A. C., Quinn, J. L., et al. 2002, ApJ, 579, 639, doi: 10.1086/342225

Bennett, D. P., Bhattacharya, A., Anderson, J., et al. 2015, ApJ, 808, 169, doi: 10.1088/0004-637X/808/2/169

Bird, S., Cholis, I., Muñoz, J. B., et al. 2016, Physical Review Letters, 116, 201301, doi: 10.1103/PhysRevLett.116.201301

Bovy, J., Leung, H. W., Hunt, J. A. S., et al. 2019, arXiv e-prints, arXiv:1905.11404. https://arxiv.org/abs/1905.11404

Bullock, J. S., \& Johnston, K. V. 2005, ApJ, 635, 931, doi: $10.1086 / 497422$

Campos, F., Bergeron, P., Romero, A. D., et al. 2016, MNRAS, 456, 3729, doi: 10.1093/mnras/stv2911

Carr, B., Kühnel, F., \& Sandstad, M. 2016, Phys. Rev. D, 94, 083504, doi: 10.1103/PhysRevD.94.083504

Castelli, F., \& Kurucz, R. L. 2004, ArXiv Astrophysics e-prints

Chapline, G., \& Barbieri, J. 2018, LHEP, 1, 17, doi: 10.31526/LHEP.1.2018.04

Choi, J., Dotter, A., Conroy, C., et al. 2016, ApJ, 823, 102, doi: 10.3847/0004-637X/823/2/102

Clarke, J. P., Wegg, C., Gerhard, O., et al. 2019, MNRAS, 489, 3519, doi: 10.1093/mnras/stz2382

Clarkson, W., Sahu, K., Anderson, J., et al. 2008, ApJ, 684, 1110, doi: 10.1086/590378

Clarkson, W. I., Calamida, A., Sahu, K. C., et al. 2018, ApJ, 858, 46, doi: 10.3847/1538-4357/aaba7f

Dai, S., Smith, M. C., Lin, M. X., et al. 2015, ApJ, 802, 120, doi: 10.1088/0004-637X/802/2/120

Damineli, A., Almeida, L. A., Blum, R. D., et al. 2016, MNRAS, 463, 2653, doi: 10.1093/mnras/stw2122

Di Stefano, R., \& Esin, A. A. 1995, ApJL, 448, L1, doi: 10.1086/309588

Dominik, M. 1998, A\&A, 333, 893. https://arxiv.org/abs/astro-ph/9801119

-. 2009, MNRAS, 393, 816, doi: 10.1111/j.1365-2966.2008.14276.x

Dominik, M., \& Sahu, K. C. 2000, ApJ, 534, 213, doi: $10.1086 / 308716$
Dong, S., Mérand, A., Delplancke-Ströbele, F., et al. 2019, ApJ, 871, 70, doi: 10.3847/1538-4357/aaeffb

Dotter, A. 2016, ApJS, 222, 8, doi: $10.3847 / 0067-0049 / 222 / 1 / 8$

Drlica-Wagner, A., Mao, Y.-Y., Adhikari, S., et al. 2019, arXiv e-prints. https://arxiv.org/abs/1902.01055

Duchêne, G., \& Kraus, A. 2013, ARA\&A, 51, 269, doi: 10.1146/annurev-astro-081710-102602

Elbert, O. D., Bullock, J. S., \& Kaplinghat, M. 2018, MNRAS, 473, 1186, doi: 10.1093/mnras/stx1959

Farr, W. M., Sravan, N., Cantrell, A., et al. 2011, ApJ, 741, 103, doi: 10.1088/0004-637X/741/2/103

Fryer, C. L., Belczynski, K., Wiktorowicz, G., et al. 2012, ApJ, 749, 91, doi: 10.1088/0004-637X/749/1/91

Gaia Collaboration, Prusti, T., de Bruijne, J. H. J., et al. 2016, A\&A, 595, A1, doi: 10.1051/0004-6361/201629272

Gaudi, B. S. 2012, ARA\&A, 50, 411, doi: 10.1146/annurev-astro-081811-125518

Green, G. M., Schlafly, E. F., Finkbeiner, D., et al. 2018, MNRAS, 478, 651, doi: 10.1093/mnras/sty1008

Han, C. 1999, MNRAS, 309, 373, doi: 10.1046/j.1365-8711.1999.02832.x

Han, C., \& Gould, A. 2003, ApJ, 592, 172, doi: $10.1086 / 375706$

Han, C., \& Jeong, Y. 1998, MNRAS, 301, 231, doi: 10.1046/j.1365-8711.1998.02023.x

Heger, A., Fryer, C. L., Woosley, S. E., Langer, N., \& Hartmann, D. H. 2003, ApJ, 591, 288, doi: $10.1086 / 375341$

Hosek, Matthew W., J., Lu, J. R., Anderson, J., et al. 2019, ApJ, 870, 44, doi: 10.3847/1538-4357/aaef90

Howard, C. D., Rich, R. M., Reitzel, D. B., et al. 2008, ApJ, 688, 1060, doi: 10.1086/592106

Howard, C. D., Rich, R. M., Clarkson, W., et al. 2009, ApJL, 702, L153, doi: 10.1088/0004-637X/702/2/L153

Hunter, J. D. 2007, Computing in Science \& Engineering, 9, 90, doi: 10.1109/MCSE.2007.55

Husser, T.-O., Wende-von Berg, S., Dreizler, S., et al. 2013, A\&A, 553, A6, doi: 10.1051/0004-6361/201219058

Janka, H.-T. 2012, Annual Review of Nuclear and Particle Science, 62, 407, doi: 10.1146/annurev-nucl-102711-094901

Janka, H.-T. 2013, Monthly Notices of the Royal Astronomical Society, 434, 1355, doi: $10.1093 / \mathrm{mnras} / \mathrm{stt} 1106$

Kalirai, J. S., Hansen, B. M. S., Kelson, D. D., et al. 2008, ApJ, 676, 594, doi: 10.1086/527028

Kerins, E., Robin, A. C., \& Marshall, D. J. 2009, MNRAS, 396, 1202, doi: 10.1111/j.1365-2966.2009.14791.x 
Kroupa, P. 2001, MNRAS, 322, 231, doi: 10.1046/j.1365-8711.2001.04022.x

Lai, D. 2001, in Lecture Notes in Physics, Berlin Springer Verlag, Vol. 578, Physics of Neutron Star Interiors, ed. D. Blaschke, N. K. Glendenning, \& A. Sedrakian, 424

Lu, J. R., Sinukoff, E., Ofek, E. O., Udalski, A., \& Kozlowski, S. 2016, ApJ, 830, doi: 10.3847/0004-637X/830/1/41

Maeda, Y., Kubota, A., Kobayashi, Y., et al. 2005, ApJL, 631, L65, doi: 10.1086/491698

Mao, S., \& Paczynski, B. 1991, ApJL, 374, L37, doi: 10.1086/186066

Mao, S., Smith, M. C., Woźniak, P., et al. 2002, MNRAS, 329, 349, doi: 10.1046/j.1365-8711.2002.04986.x

Margalit, B., \& Metzger, B. D. 2017, ApJL, 850, L19, doi: $10.3847 / 2041-8213 /$ aa991c

Mashian, N., \& Loeb, A. 2017, MNRAS, 470, 2611, doi: 10.1093/mnras/stx1410

Mróz, P., Udalski, A., Skowron, J., et al. 2017, Nature, 548, 183, doi: 10.1038/nature23276

—. 2019, ApJS, 244, 29, doi: 10.3847/1538-4365/ab426b

Muraki, Y., Sumi, T., Abe, F., et al. 1999, Progress of Theoretical Physics Supplement, 133, 233, doi: 10.1143/PTPS.133.233

Nataf, D. M., Gould, A., Fouqué, P., et al. 2013, ApJ, 769, 88, doi: 10.1088/0004-637X/769/2/88

Nucita, A. A., De Paolis, F., Ingrosso, G., et al. 2006, ApJ, 651, 1092, doi: 10.1086/507784

Nucita, A. A., De Paolis, F., Ingrosso, G., Giordano, M., \& Manni, L. 2016, ApJ, 823, 120, doi: 10.3847/0004-637X/823/2/120

Osłowski, S., Moderski, R., Bulik, T., \& Belczynski, K. 2008, A\&A, 478, 429, doi: 10.1051/0004-6361:20066802

Özel, F., \& Freire, P. 2016, ARA\&A, 54, 401, doi: 10.1146/annurev-astro-081915-023322

Özel, F., Psaltis, D., Narayan, R., \& McClintock, J. E. 2010, ApJ, 725, 1918, doi: 10.1088/0004-637X/725/2/1918

Paczynski, B. 1986, ApJ, 304, 1, doi: 10.1086/164140

Paxton, B., Bildsten, L., Dotter, A., et al. 2011, ApJS, 192, 3, doi: 10.1088/0067-0049/192/1/3

Paxton, B., Cantiello, M., Arras, P., et al. 2013, ApJS, 208, 4, doi: 10.1088/0067-0049/208/1/4

Paxton, B., Marchant, P., Schwab, J., et al. 2015, ApJS, 220, 15, doi: 10.1088/0067-0049/220/1/15

Penny, M. T., Gaudi, B. S., Kerins, E., et al. 2019, The Astrophysical Journal Supplement Series, 241, 3, doi: 10.3847/1538-4365/aafb69

Penny, M. T., Kerins, E., Rattenbury, N., et al. 2013, MNRAS, 434, 2, doi: 10.1093/mnras/stt927
Pflamm-Altenburg, J., \& Kroupa, P. 2006, MNRAS, 373, 295, doi: 10.1111/j.1365-2966.2006.11028.x

Poindexter, S., Afonso, C., Bennett, D. P., et al. 2005, ApJ, 633, 914, doi: 10.1086/468182

Portail, M., Gerhard, O., Wegg, C., \& Ness, M. 2017, MNRAS, 465, 1621, doi: 10.1093/mnras/stw2819

Raithel, C. A., Sukhbold, T., \& Özel, F. 2018, ApJ, 856, 35, doi: 10.3847/1538-4357/aab09b

Remillard, R. A., \& McClintock, J. E. 2006, ARA\&A, 44, 49, doi: 10.1146/annurev.astro.44.051905.092532

Repetto, S., Davies, M. B., \& Sigurdsson, S. 2012, MNRAS, 425, 2799, doi: 10.1111/j.1365-2966.2012.21549.x

Repetto, S., Igoshev, A. P., \& Nelemans, G. 2017, MNRAS, 467, 298, doi: 10.1093/mnras/stx027

Robin, A. C., Marshall, D. J., Schultheis, M., \& Reylé, C. 2012, A\&A, 538, A106, doi: 10.1051/0004-6361/201116512

Robin, A. C., Reylé, C., Derrière, S., \& Picaud, S. 2003, A\&A, 409, 523, doi: 10.1051/0004-6361:20031117

Rybicki, K. A., Wyrzykowski, Ł., Klencki, J., et al. 2018, MNRAS, 476, 2013, doi: 10.1093/mnras/sty356

Sahu, K. C., Anderson, J., Casertano, S., et al. 2017, Science, 356, 1046, doi: 10.1126/science.aal2879

Sanders, J. L., Smith, L., \& Evans, N. W. 2019, MNRAS, 1855, doi: 10.1093/mnras/stz1827

Sasaki, M., Suyama, T., Tanaka, T., \& Yokoyama, S. 2016, Physical Review Letters, 117, 061101, doi: 10.1103/PhysRevLett.117.061101

Schlegel, D. J., Finkbeiner, D. P., \& Davis, M. 1998, ApJ, 500, 525, doi: 10.1086/305772

Sharma, S., Bland-Hawthorn, J., Johnston, K. V., \& Binney, J. 2011, ApJ, 730, 3, doi: $10.1088 / 0004-637 \mathrm{X} / 730 / 1 / 3$

Shu, F. H. 1969, ApJ, 158, 505, doi: 10.1086/150214

Smith, M. C., Mao, S., Woźniak, P., et al. 2002, MNRAS, 336, 670, doi: 10.1046/j.1365-8711.2002.05811.x

Spera, M., Mapelli, M., \& Bressan, A. 2015, MNRAS, 451, 4086, doi: 10.1093/mnras/stv1161

Spergel, D., Gehrels, N., Baltay, C., et al. 2015, arXiv e-prints, arXiv:1503.03757. https://arxiv.org/abs/1503.03757

Sukhbold, T., Ertl, T., Woosley, S. E., Brown, J. M., \& Janka, H.-T. 2016, ApJ, 821, 38, doi: 10.3847/0004-637X/821/1/38

Sukhbold, T., Woosley, S. E., \& Heger, A. 2018, ApJ, 860, 93, doi: 10.3847/1538-4357/aac2da

Sumi, T., \& Penny, M. T. 2016, ApJ, 827, 139, doi: 10.3847/0004-637X/827/2/139

Sumi, T., Kamiya, K., Bennett, D. P., et al. 2011, Nature, 473, 349, doi: 10.1038/nature10092 
Sumi, T., Bennett, D. P., Bond, I. A., et al. 2013, ApJ, 778, 150, doi: 10.1088/0004-637X/778/2/150

The LIGO Scientific Collaboration, the Virgo Collaboration, Abbott, B. P., et al. 2018, arXiv e-prints, arXiv:1811.12940. https://arxiv.org/abs/1811.12940

Timmes, F. X., Woosley, S. E., \& Weaver, T. A. 1996, ApJ, 457, 834, doi: 10.1086/176778

Tisserand, P., Le Guillou, L., Afonso, C., et al. 2007, A\&A, 469, 387, doi: 10.1051/0004-6361:20066017

Udalski, A., Szymanski, M., Kaluzny, J., Kubiak, M., \& Mateo, M. 1992, AcA, 42, 253

Udalski, A., Szymański, M. K., \& Szymański, G. 2015, AcA, 65, 1. https://arxiv.org/abs/1504.05966

Udalski, A., Szymanski, M., Stanek, K. Z., et al. 1994, AcA, 44, 165. https://arxiv.org/abs/astro-ph/9407014

van der Walt, S., Colbert, S. C., \& Varoquaux, G. 2011, Computing in Science \& Engineering, 13, 22, doi: 10.1109/MCSE.2011.37

Virtanen, P., Gommers, R., Oliphant, T. E., et al. 2019, arXiv e-prints, arXiv:1907.10121.

https://arxiv.org/abs/1907.10121
Wegg, C., \& Gerhard, O. 2013, MNRAS, 435, 1874, doi: $10.1093 / \mathrm{mnras} / \mathrm{stt} 1376$

Wegg, C., Gerhard, O., \& Portail, M. 2015, MNRAS, 450, 4050, doi: 10.1093/mnras/stv745

Wiktorowicz, G., Wyrzykowski, Ł., Chruslinska, M., et al. 2019, arXiv e-prints, arXiv:1907.11431.

https://arxiv.org/abs/1907.11431

Wood, A., \& Mao, S. 2005, MNRAS, 362, 945, doi: 10.1111/j.1365-2966.2005.09357.x

Woźniak, P., \& Paczyński, B. 1997, ApJ, 487, 55, doi: 10.1086/304607

Wyrzykowski, Ł., \& Mandel, I. 2019, arXiv e-prints, arXiv:1904.07789. https://arxiv.org/abs/1904.07789

Wyrzykowski, L., Skowron, J., Kozłowski, S., et al. 2011, MNRAS, 416, 2949, doi: 10.1111/j.1365-2966.2011.19243.x

Wyrzykowski, Ł., Rynkiewicz, A. E., Skowron, J., et al. 2015, ApJS, 216, 12, doi: 10.1088/0067-0049/216/1/12

Wyrzykowski, Ł., Kostrzewa-Rutkowska, Z., Skowron, J., et al. 2016, MNRAS, 458, 3012, doi: $10.1093 / \mathrm{mnras} / \mathrm{stw} 426$ 